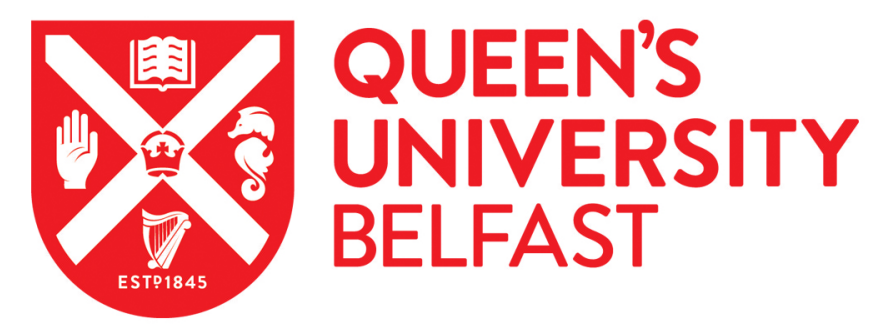

\title{
Superluminous Supernovae as Standardizable Candles and High- Redshift Distance Probes
}

Inserra, C., \& Smartt, S. J. (2014). Superluminous Supernovae as Standardizable Candles and High-Redshift Distance Probes. Astrophysical Journal, 796(2), [87]. https://doi.org/10.1088/0004-637X/796/2/87

Published in:

Astrophysical Journal

Document Version:

Peer reviewed version

Queen's University Belfast - Research Portal:

Link to publication record in Queen's University Belfast Research Portal

Publisher rights

(c) 2014. The American Astronomical Society. All rights reserved.

\section{General rights}

Copyright for the publications made accessible via the Queen's University Belfast Research Portal is retained by the author(s) and / or other copyright owners and it is a condition of accessing these publications that users recognise and abide by the legal requirements associated with these rights.

Take down policy

The Research Portal is Queen's institutional repository that provides access to Queen's research output. Every effort has been made to ensure that content in the Research Portal does not infringe any person's rights, or applicable UK laws. If you discover content in the Research Portal that you believe breaches copyright or violates any law, please contact openaccess@qub.ac.uk. 


\title{
SUPERLUMINOUS SUPERNOVAE AS STANDARDIZABLE CANDLES AND HIGH-REDSHIFT DISTANCE PROBES
}

\author{
C. INSERRA AND S. J. SMARTT \\ Astrophysics Research Centre, School of Mathematics and Physics, Queens University Belfast, Belfast BT7 1NN, UK; c.inserra@qub.ac.uk \\ Received 2014 July 7; accepted 2014 September 28; published 2014 November 10
}

\begin{abstract}
We investigate the use of type Ic superluminous supernovae (SLSN Ic) as standardizable candles and distance indicators. Their appeal as cosmological probes stems from their remarkable peak luminosities, hot blackbody temperatures, and bright rest-frame ultraviolet emission. We present a sample of 16 published SLSN, from redshifts 0.1 to 1.2 , and calculate accurate $K$ corrections to determine uniform magnitudes in 2 synthetic rest-frame filter bandpasses with central wavelengths at $400 \mathrm{~nm}$ and $520 \mathrm{~nm}$. At $400 \mathrm{~nm}$, we find an encouragingly low scatter in their uncorrected, raw mean magnitudes with $M(400)=-21.86 \pm 0.35$ mag for the full sample of 16 objects. We investigate the correlation between their decline rates and peak magnitude and find that the brighter events appear to decline more slowly. In a manner similar to the Phillips relation for type Ia SNe (SNe Ia), we define a $\Delta M_{20}$ decline relation. This correlates peak magnitude and decline over 20 days and can reduce the scatter in standardized peak magnitudes to $\pm 0.22 \mathrm{mag}$. We further show that $M(400)$ appears to have a strong color dependence. Redder objects are fainter and also become redder faster. Using this peak magnitudecolor evolution relation, a surprisingly low scatter of between $\pm 0.08 \mathrm{mag}$ and $\pm 0.13 \mathrm{mag}$ can be found in peak magnitudes, depending on sample selection. However, we caution that only 8 to 10 objects currently have enough data to test this peak magnitudecolor evolution relation. We conclude that SLSN Ic are promising distance indicators in the high-redshift universe in regimes beyond those possible with SNe Ia. Although the empirical relationships are encouraging, the unknown progenitor systems, how they may evolve with redshift, and the uncertain explosion physics are of some concern. The two major measurement uncertainties are the limited numbers of low-redshift, well-studied objects available to test these relationships and internal dust extinction in the host galaxies.
\end{abstract}

Key words: distance scale - supernovae: general

Online-only material: color figures

\section{INTRODUCTION}

In recent years, the new generation of deep, wide surveys like the Palomar Transient Factory (PTF; Rau et al. 2009), the Panoramic Survey Telescope \& Rapid Response System (Pan-STARRS; Kaiser et al. 2010), the Catalina Real-time Transient Survey (CRTS; Drake et al. 2009), and the La Silla QUEST survey (Baltay et al. 2013) have searched for supernovae $(\mathrm{SNe})$ in the local universe without a galaxy bias and have found new types of luminous transients. These were preceded by the smaller scale, but pioneering, Texas Supernova Search (TSS; Quimby et al. 2005), which uncovered the first SN recognized as "superluminous" (Quimby 2006; Quimby et al. 2007; Smith et al. 2007). These superluminous supernovae (hereafter SLSN) are characterized by absolute magnitudes at maximum light of $M_{\mathrm{AB}}<-21 \mathrm{mag}$ and total radiated energies of the order of $10^{51} \mathrm{erg}$. They are factors of 5-100 brighter than type Ia $\mathrm{SNe}$ (SNe Ia) or normal core-collapse events. In reviewing the discoveries to date, Gal-Yam (2012) proposed three observational classes for these highly luminous $\mathrm{SNe}$, SLSN I, SLSN II, and SLSN R, based on their photometric evolution and presumed physical characteristics.

The SLSN I are hydrogen-free and show a blue continuum, a distinctive "W"-shaped spectral feature at $\sim 4200 \AA$, and a transformation in their spectra to those of normal type Ic SNe. They were first recognized by Quimby et al. (2011b), who tied the transients SN2005ap and SCP-06F6 (Quimby et al. 2007; Barbary et al. 2009) together with PTF discoveries by determining their redshifts and common broad photospheric absorption lines. A detailed study of one of the nearer events
(SN2010gx/PTF10cwr at $z=0.23$ ) by Pastorello et al. (2010a) showed that it spectroscopically evolved into a type Ic SNe but on much longer timescales than usual. The possibility of quantitatively studying these at much higher redshifts $(z \sim 1)$ than the known SN population was shown by Chomiuk et al. (2011) in the Pan-STARRS1 Medium Deep field survey. Inserra et al. (2013) recently reported a detailed study of a sample of five nearby SLSN and explored quantitative models to fit the light curves. They favored a model of a spinning-down magnetic neutron star as the source of the energy input to power their luminous light curves. This magnetar model for luminous $\mathrm{SNe}$ was theoretically proposed by Kasen \& Bildsten (2010), Woosley (2010), and further investigated by Dessart et al. (2012). Because the optical spectra of these SNe evolve to be similar to the normal and more common classes of type Ic SNe, Inserra et al. (2013) simply termed them SLSN Ic.

Gal-Yam (2012) suggested a separate definition of "SLSN R" mostly based on the observed slow light curve evolution of SN2007bi (Gal-Yam et al. 2009; Young et al. 2010) and the fact that this slow decline could be explained theoretically by the decay of radioactive ${ }^{56} \mathrm{Ni}$ and ${ }^{56} \mathrm{Co}$ (hence the use of "R"). However, recent studies by Nicholl et al. (2013) and McCrum et al. (2014a) showed that two SLSN that are very similar to SN2007bi are better explained by the magnetar model. In fact, the physical nature of the radioactive powering is not unambiguously established in any of them, which means that the label of "SLSN R" is not one that is observationally secure. Nicholl et al. (2013) illustrated the similarity in the early spectra of the SLSN I types (of Pastorello et al. 2010a; Quimby et al. 2011b; Inserra et al. 2013) and the SN2007bi-like 
events (PTF12dam in particular). It is certainly possible that the "SLSN I" and the "SLSN R" explosions have the same physical mechanism. However, it is not clear yet if there is a continuum in their decay timescales or if there are two distinct groups that decline with different rates after peak. Following Inserra et al. (2013) and Nicholl et al. (2013), we will use the classification term SLSN Ic to refer to all of the hydrogen-poor SLSNe. Their physical nature may be debated, but the simple observational term of type Ic is quite secure in the classical scheme of SN classification. In order to distinguish between the two observational groups, we will use the terms SN2005ap-like and SN2007bi-like events because these are the prototypes of the faster and slower evolving events known to date.

The SLSN of type II generally show strong Hi lines in emission, which indicate the interaction of fast-moving ejecta with a preexisting and slower moving shell of material. In this scenario, the kinetic energy of the ejecta (with energies of the order of $10^{51} \mathrm{erg}$ ) is converted to luminosity during the collision (e.g., Smith \& McCray 2007; Agnoletto et al. 2009). These have been relatively easy to identify due to the strong emission line signatures, although there is still uncertain physics concerning the symmetry and clumping of the interacting shells. The interaction model has been extended to explain the hydrogenfree SLSN by Chevalier \& Irwin (2011) and Ginzburg \& Balberg (2012). Benetti et al. (2014) have recently shown that the transient CSS121015:004244+132827 appears quite similar to many of the SLSN Ic of Quimby et al. (2011b) and Inserra et al. (2013), but weak and transitory $\mathrm{H} \alpha$ emission is detected, suggestive of interaction occurring.

The number of SLSN Ic that have been discovered and recognized as such has increased rapidly, with discoveries out to redshifts of $z \sim 4$ (Chomiuk et al. 2011; Cooke et al. 2012; Berger et al. 2012; Leloudas et al. 2012; Inserra et al. 2012; Lunnan et al. 2013; Howell et al. 2013; Kostrzewa-Rutkowska et al. 2013; Benetti et al. 2014; McCrum et al. 2014b). They seem to be fairly homogeneous in their spectroscopic and photometric properties and have attracted considerable attention because of their potential utility as cosmological standard candles. The potential lies in the fact that they are 2-3 mag brighter than $\mathrm{SNe}$ Ia, and their hot blackbody temperatures mean that their UV continuum emission is detectable at high redshifts in the optical and near-infrared (e.g., Cooke et al. 2012). The study of these objects is still a relatively youthful endeavor because they were first recognized as highly luminous in 2010-2011 (Pastorello et al. 2010a; Quimby et al. 2011b; Chomiuk et al. 2011). Quimby et al. (2013b) already pointed out that the peak magnitudes of SLSN Ic may be quite narrowly distributed at $M=-21.7 \pm 0.4$ (in the unfiltered ROTSE-IIIb bandpass). A detailed investigation of this issue is now possible with a large sample of well-observed SLSN Ic, which have multicolor light curves and spectral sequences to allow more accurate $K$ corrections to be determined. King et al. (2014) discussed how novel high-redshift standard candles could be used to probe the dark energy equation of state $w(z)$ and how this compares to the current state-of-the-art SN Ia and baryon acoustic oscillation measurements. It would appear that a large number $\left(\sim 10^{3}\right)$ are required to improve on $w(z)$ estimates, and objects should cover a long redshift baseline, which is challenging. Nevertheless, any additional cosmological probes are potentially useful, and their merits should be studied.

In this paper we analyze a sample of well-observed SLSN Ic with complete light curves from before peak to beyond 30 days. We test whether there is any relation between the empirical, observable quantities (such as light curve shape, color evolution, and peak luminosity) that could make them useful cosmological standardizable candles. It is well known that the absolute magnitudes of SNe Ia have been standardized using different methods based on their empirical characteristics. The earliest and simplest standardization method correlated peak magnitudes with decline rates and is referred to as the $\Delta m_{15}$ method (Rust 1974; Pskovskii 1977; Phillips 1993; Hamuy et al. 1996). This gave way to much more sophisticated approaches, such as the multicolor light curve shapes method (Riess et al. 1996, 1998), the stretch method (Perlmutter et al. 1997; Goldhaber et al. 2001), the spectral adaptive light curve template (SALT) method (Guy et al. 2005, 2007), and the BayeSN method (Mandel et al. 2009, 2011). In the context of this initial attempt to standardize SLSN Ic, it is useful to reflect on the benchmark results from the early attempts with the $\Delta m_{15}$ method. Phillips (1993) used nine SNe and illustrated the large dispersion in the raw absolute magnitudes (without any correction) of $\sigma=0.8,0.6$, and 0.5 for $B, V$, and $I$, respectively. ${ }^{1}$ The Phillips $\Delta m_{15}$ correlation immediately reduced the scatter to $\sigma=0.36,0.28$, and 0.38 in $B V I$. This method was revisited with a much larger sample by Prieto et al. (2006), who produced an rms scatter around the linear fit of $\sigma=$ $0.17,0.14$ for $94 \mathrm{SNe}$ in $B V$ and 0.15 for $87 \mathrm{SNe}$ in the $I$ band. Since that time, the community has been investigating other standardizable candles with a view to reaching similar scatter values. Type II-P SNe have been proposed, which appear to have an rms scatter in their standardizable luminosity-velocity relation of around 0.26 mag (Hamuy \& Pinto 2002; Nugent et al. 2006; Poznanski et al. 2009; D’Andrea et al. 2010). Maguire et al. (2010) suggested that going to the near-infrared (NIR) could potentially reduce this to $0.1-0.15 \mathrm{mag}$, although the disadvantage of SNe II-P as distance indicators is that they are 2-3 mag fainter than SNe Ia. We note that GRB have shown potential to be standardizable candle (Cano \& Jakobsson 2014; Cano 2014). These attempts illustrate that achieving an rms scatter of $0.1-0.3 \mathrm{mag}$ for any new standardizable candle would make it competitive and interesting to study further. This paper investigates the use of SLSN Ic as such standardizable candles.

\section{THE SAMPLE AND DATA}

The sample chosen was selected from the published SLSN Ic that have well-sampled light curves around peak luminosity. We required photometric coverage from several days premaximum (or a good estimate of the time and value of peak luminosity) to about 35 rest-frame days after peak luminosity. We further required photometry in observer frame filters that, after $K$ correction, had similar equivalent rest-frame bandpasses (see Section 4). An accurate spectroscopic redshift is also required to provide reliable relative distances. The redshift distribution of the known population of well-observed SLSN $(0.1 \leqslant z \leqslant$ 1.6) means that this selection is not trivial, and not all of the 30-40 or so known SLSN can be used in our sample calculations (see Lunnan et al. 2014 for an extensive, but not complete, compilation of $31 \mathrm{SLSN}$ ). We found $16 \mathrm{SNe}$ that had suitable data for our analysis: SN2010gx, SN2011kf, SN2011ke, SN2012il, SN2005ap, PS1-10ky, PTF09cnd, SCP06F6, PTF11rks, PS1-10bzj, PS1-10pm, LSQ12dlf, SN2013dg, PS1-11ap, PTF12dam, and CSS121015:004244+132827 (with

\footnotetext{
1 The $\sigma$ for the raw absolute magnitudes is simply the standard deviation of the population, as calculated by Phillips (1993). The $\sigma$ or scatter around the best-fit line for $\Delta m_{15}$ is the rms error of the linear fit.
} 
Table 1

Sample of SLSN

\begin{tabular}{|c|c|c|c|c|c|c|c|c|c|c|c|}
\hline $\mathrm{SN}$ & $z$ & $\begin{array}{c}E \\
(B-V)\end{array}$ & $m$ & Ref. $^{\mathrm{a}}$ & Filters 400, 520 & $A_{400}$ & $\begin{array}{c}M \\
(400)\end{array}$ & $\begin{array}{l}\Delta M_{10} \\
(400)\end{array}$ & $\begin{array}{l}\Delta M_{20} \\
(400)\end{array}$ & $\begin{array}{l}\Delta M_{30} \\
(400)\end{array}$ & $\begin{array}{c}\Delta M \\
(400-520)_{30}\end{array}$ \\
\hline \multicolumn{12}{|c|}{ SLSN Ic (2005ap-like) } \\
\hline SN2011ke & 0.143 & 0.01 & $17.70(g)$ & 1 & $g \rightarrow 400, r \rightarrow 520$ & 0.05 & -21.65 & 0.49 & 1.33 & 2.47 & 0.54 \\
\hline SN2012il & 0.175 & 0.02 & $18.00(g)$ & 1 & $g \rightarrow 400, r \rightarrow 520$ & 0.09 & -21.77 & 0.49 & 1.33 & 2.19 & 0.49 \\
\hline PTF11rks & 0.190 & 0.04 & $19.13(g)$ & 1 & $g \rightarrow 400, r \rightarrow 520$ & 0.17 & -20.97 & 0.44 & 1.78 & 2.62 & 0.95 \\
\hline SN2010gx & 0.230 & 0.04 & $18.43(g)$ & 2 & $g \rightarrow 400, r \rightarrow 520$ & 0.13 & -21.93 & 0.33 & 1.01 & 2.00 & 0.34 \\
\hline SN2011kf & 0.245 & 0.02 & $18.60(g)$ & 1 & $g \rightarrow 400$ & 0.09 & -21.91 & 0.14 & 0.68 & 1.49 & $\ldots$ \\
\hline LSQ12dlf & 0.255 & 0.01 & $18.78(V)$ & 3 & $V \rightarrow 400, r \rightarrow 520$ & 0.03 & -22.02 & 0.45 & 0.54 & 1.00 & 0.29 \\
\hline PTF09cnd & 0.258 & 0.03 & $18.29(R)$ & 4 & $r \rightarrow 400$ & 0.05 & -22.35 & 0.19 & 0.60 & 1.09 & $\cdots$ \\
\hline SN2013dg & 0.265 & 0.01 & $19.06(g)$ & 3 & $g \rightarrow 400, r \rightarrow 520$ & 0.03 & -21.81 & 0.50 & 1.26 & 2.08 & 0.51 \\
\hline SN2005ap & 0.283 & 0.01 & $18.35(R)$ & 5 & $R \rightarrow 400$ & 0.02 & -21.66 & 0.22 & 0.75 & $\cdots$ & $\cdots$ \\
\hline PS1-10bzj & 0.650 & 0.01 & $21.23(r)$ & 6 & $R \rightarrow 400, z \rightarrow 520$ & 0.02 & -21.40 & 0.30 & 0.96 & 1.70 & 0.60 \\
\hline PS1-10ky & 0.956 & 0.03 & $21.15(i)$ & 7 & $i \rightarrow 400, z \rightarrow 520$ & 0.06 & -21.88 & 0.10 & 0.65 & 1.31 & 0.30 \\
\hline SCP-06F6 & 1.189 & 0.01 & $21.04(z)$ & 8 & $z \rightarrow 400$ & 0.01 & -22.14 & 0.20 & 0.50 & 0.89 & $\ldots$ \\
\hline PS1-10pm & 1.206 & 0.02 & $21.23(z)$ & 9 & $z \rightarrow 400$ & 0.02 & -22.01 & 0.12 & 0.44 & $\cdots$ & $\cdots$ \\
\hline \multicolumn{12}{|c|}{ SLSN Ic (2007bi-like) } \\
\hline PTF12dam & 0.107 & 0.01 & $16.57(g)$ & 10 & $g \rightarrow 400, r \rightarrow 520$ & 0.04 & -21.89 & 0.12 & 0.23 & 0.48 & 0.07 \\
\hline PS1-11ap & 0.524 & 0.01 & $20.20(r)$ & 11 & $r \rightarrow 400, i \rightarrow 520$ & 0.02 & -21.87 & 0.02 & 0.14 & 0.16 & 0.02 \\
\hline \multicolumn{12}{|c|}{ SLSN II } \\
\hline CSS121015 & 0.287 & 0.08 & $18.58(V)$ & 12 & $V \rightarrow 400, R \rightarrow 520$ & 0.24 & -22.46 & 0.25 & 0.76 & 0.93 & 0.21 \\
\hline
\end{tabular}

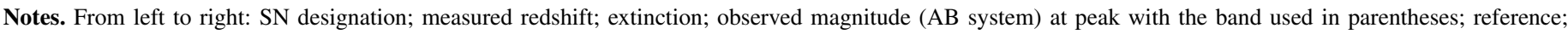

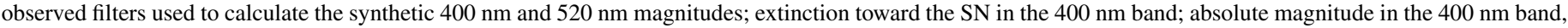
magnitude decrease in 10, 20, and 30 days; the color change between the $400 \mathrm{~nm}$ and $520 \mathrm{~nm}$ synthetic bands at peak and 30 days after.

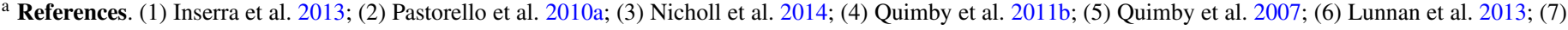
Chomiuk et al. 2011; (8) Barbary et al. 2009; (9) McCrum et al. 2014b; (10) Nicholl et al. 2013; (11) McCrum et al. $2014 \mathrm{a}$; (12) Benetti et al. 2014.

data from Pastorello et al. 2010a; Inserra et al. 2013; Quimby et al. 2007, 2011b; Barbary et al. 2009; Chomiuk et al. 2011; Lunnan et al. 2013; Nicholl et al. 2013, 2014; McCrum et al. 2014a, 2014b; Benetti et al. 2014). The details are listed in Table 1. We highlight the important caveat that this sample selection does not take a potential Malmquist bias into account. The identification process of these targets comes from a range of surveys, each with quite different magnitude limits combined with different selection procedures for selecting targets for spectroscopic follow-up. Hence the process from discovery of a transient to identification as an SLSN is quite inhomogeneous. It is certainly true that the intrinsically brightest $\mathrm{SNe}$ are overrepresented in magnitude-limited surveys compared to their volumetric rates, which may introduce a shift in absolute magnitude simply because the lower luminosity events are underrepresented. There does appear to be a significant gap between the SLSN Ic and the brightest (and most energetic) of type Ic SNe (e.g., see Inserra et al. 2013 for a comparison). Whether this gap is a Malmquist-type bias or real remains to be investigated.

Thirteen of these objects appear to be similar to the wellobserved SN2010gx, and these decay rapidly after peak magnitude. The similarity of their light curves and spectral evolution has been discussed by Quimby et al. (2011b), Inserra et al. (2013), and Gal-Yam (2012), amongst others, and we label these as 2005ap-like because that was the first one discovered. Of our sample of 16, 2 (PTF12dam and PS1-11ap) decline on noticeably slower timescales and are similar to SN2007bi (see Section 1). We include them in our analysis for completeness and show results with and without these two events (and refer to them as 2007bi-like). The object CSS121015:004244+132827 (hereafter CSS121015; Benetti et al. 2014) has been classified as a SLSN II because it shows signs of $\mathrm{H} \alpha$ that is probably linked to the SN rather than the host galaxy. We include it here for completeness because its spectra resemble SN2010gx and the other SLSN Ic with the addition of weak hydrogen features, and at high redshift one might struggle to see the subtle differences present in the spectra. Again, we will show results with and without this event. We note that although we included it and label it an SLSN II, we are not considering the broader and more populous class of SLSN II, such as the SN2006gy-like events. These luminous type IIn SNe are unambiguously driven by strong circumstellar material interaction and collisions of hydrogen-rich gas, and they are observationally very distinct. Hereafter we will refer to three samples. The 13 2005ap-like events are called the "pure sample." Including the 2 2007bi-like objects makes an "extended sample" of 15 objects, and adding in CSS121015 defines the "complete sample" of 16 objects.

We included all SLSN having enough data to be useful for our analysis. The SLSN published in the literature that are not reported and used here were rejected primarily because they lack the photometric data required for our careful and uniform analysis. There are at least seven other SLSN Ic that are securely classified in previous work and have data published, but their photometric coverage does not present a complete enough data set around the epoch of 0-30 days (rest frame) in filters that are suitable for the comparison we undertake in Section 4. These are SN2006oz (Leloudas et al. 2012), PTF09cwl and PTF09atu (Quimby et al. 2011b), PTF10hgi (Inserra et al. 2013), PS1-10awh (Chomiuk et al. 2011), PS1-11bam (Berger et al. 2012), and SSS120810:231802-560926 (Nicholl et al. 2014). We did not consider PS1-10afx further because it is probably a lensed SN Ia and not an SLSN (Chornock et al. 2013; Quimby et al. 2013a, 2014). We also did not include the slowly fading SLSN SN2007bi (Gal-Yam et al. 2009; Young et al. 2010) because it lacks extensive photometric coverage around the 
peak in the blue bands. SN2007bi is not as well sampled in standard filters compared to the excellent coverage of the phenomenologically similar events PTF12dam and PS1-11ap (Nicholl et al. 2013; McCrum et al. 2014a).

In our measurements of the absolute magnitude, we considered only the reddening values for interstellar dust in our Galaxy (see Table 1). The SNe of the sample were located in faint, dwarf galaxies and were unlikely to have suffered significant additional extinction. Indeed, no absorption due to $\mathrm{Na}$ I $\mathrm{D}$ lines from gas in the hosts was observed in any spectrum. This does not mean that we can exclude a priori possible dust extinction from the host. The assumption that host extinction is uniformly negligible is probably the biggest uncertainty in using SLSN Ic as standard candles. In all cases a standard reddening curve was assumed with $R_{V}=A_{V} / E(B-V)=3.1$. The objects cover a range of redshifts between 0.143 and 1.206 ; hence in the next section we discuss the appropriate corrections needed for redshift effects ( $K$ correction and time dilation) to obtain the absolute rest-frame peak magnitudes.

\section{DATA ANALYSIS}

\subsection{Redshift}

We assume a cosmology with $H_{0}=72 \mathrm{~km} \mathrm{~s}^{-1} \mathrm{Mpc}^{-1}$, $\Omega_{m}=0.27$, and $\Omega_{\Lambda}=0.73$. All of the redshifts used in this paper were evaluated spectroscopically from host galaxy lines (either emission, $\mathrm{Mg}$ II absorption, or both) with the exceptions of LSQ12dlf and SN2013dg. For these two, cross-correlation with a library of SLSN spectra was employed, retrieving a typical error on the redshift of \pm 0.005 (see Nicholl et al. 2014 for details). In the cases of SN2010gx (Pastorello et al. 2010a; Quimby et al. 2011b), SN2005ap (Quimby et al. 2011b), PS1-10ky (Chomiuk et al. 2011), PTF09cnd (Quimby et al. 2011b), SCP06F6 (Quimby et al. 2011b), PS1-10bzj (Lunnan et al. 2013), and PTF11rks (Quimby et al. 2011a), we retrieved the redshifts by analyzing the narrow absorption lines of $\mathrm{Mg}$ II doublet $\lambda \lambda 2796,2803$ and compared them to those previously published. For the other three SLSN Ic, we evaluated the redshifts by the identification of host galaxy lines, such as [O II] $\lambda 3727,[\mathrm{O}$ III $] \lambda \lambda 4959,5006, \mathrm{H} \alpha$, and $\mathrm{H} \beta$ (see with those of Quimby et al. 2010; Drake et al. 2011; Quimby et al. 2011c). In the case of SN2010gx, the redshift evaluated by the host galaxy lines (Prieto et al. 2012; Pastorello et al. 2010b, 2010a) is identical to that retrieved through the analysis of $\mathrm{Mg}$ II lines (Quimby et al. 2010, 2011b), and there are no cases where there is any measurable difference between the two methods. The two 2007bi-like events (PTF12dam and PS1-11ap) show Mg II lines in absorption. Again, the redshifts determined from this doublet were checked with the emission lines of the host galaxies (as previously done by Nicholl et al. 2013; McCrum et al. 2014a). The typical error on the spectroscopic redshift is \pm 0.003 , which corresponds to uncertainties in distance moduli of $0.07,0.02$, and 0.01 at redshifts of $z=0.1,0.5$, and 1.0 , respectively.

\subsection{K Corrections and Synthetic Photometric Bands}

Photometric and time-dependent measurements of objects at significant cosmological redshifts require application of the $K$ correction and time-dilation correction in order to provide meaningful comparative rest-frame properties. The $K$ correction is essential because measurements through a filter in the observer frame detect photons from a different part of the rest-frame spectrum than the filter throughput (e.g., Oke \& Sandage 1968; Hogg et al. 2002; Blanton \& Roweis 2007). This means that an accurate determination for the $K$ correction requires a flux-calibrated spectral energy distribution (SED) that covers a wavelength range large enough for the correction to be calculated. This is ideally done with spectra, but multicolor photometric measurements can also be usefully employed to constrain the observed SED. Such $K$ corrections have been calculated since the early days of using SNe Ia as distance indicators (e.g., Hamuy et al. 1993; Kim et al. 1996; Nugent et al. 2002), and more recently large libraries of SN Ia spectra have been employed for detailed determinations (Hsiao et al. 2007). At low redshift, it is relatively straightforward to calculate corrections to observe frame filter magnitudes such that the rest-frame magnitudes are in the same filter bandpass. However, at redshifts beyond $z \gtrsim 0.2$, the de-redshifted observed filter function is often closer to another, bluer standard filter, and hence it is more useful to determine $K$ corrections to a different filter than observed ("cross-filter" $K$ corrections). For example, at $z=0.28$ the effective central wavelength of the Pan-STARRS1 $r_{\mathrm{P} 1}$ filter corresponds to the rest-frame $g_{\mathrm{P} 1}$ filter, and hence a $K$ correction to correct the observed $r_{\mathrm{P} 1}$ to rest-frame $g_{\mathrm{P} 1}$ is useful (see Tonry et al. 2012 for filter throughput details). When the observed and rest-frame filter bands are significantly misaligned, the $K$ corrections are of course more dependent on the assumed spectral template. The corrections can be computed by using either a theoretical or empirical template spectrum (or SED) at the relevant epoch. Interpolations of spectra on either side of the particular photometric epoch can be used. Nugent et al. (2002) suggested that for SN Ia the dispersion in the cross-filter $K$ corrections is less than $1 \%$ when the interpolated spectra are constrained within \pm 4 days of the epoch, whereas Hsiao et al. (2007) showed that with a similar baseline the scatter is probably higher, at $5 \%$.

Typically, $K$ corrections are calculated to provide magnitudes in rest-frame filters that are in a standard system, such as Sloan Digital Sky Survey (SDSS) ugriz, or Johnson-Cousins $U B V R I$. In order to make a meaningful comparison of the SLSN Ic absolute magnitudes, we need to correct their observed magnitudes to a single filter. While it would be possible to correct to a standard filter in a standard system, it is not clear that this is particularly useful for our purposes. If SLSN Ic are standardizable candles, then their future use is likely to be in redshift regimes beyond those probed by SNe Ia. There is little to be gained by correcting to currently defined rest-frame filters because the lack of low-redshift SLSN Ic, which have negligible $K$ corrections, renders the standard filters fairly useless. ${ }^{2}$ Instead we choose to define two synthetic rest-frame filter bandpasses to correct to.

Analysis of the SNe rest-frame spectra during the first month from the maximum light (Pastorello et al. 2010a; Quimby et al. 2011b; Chomiuk et al. 2011; Inserra et al. 2013) indicates that the region around $4000 \AA$ is continuum dominated and relatively free from the strong spectral features that could increase the photometric dispersion from object to object. To determine synthetic magnitudes at this rest-wavelength window, we built the synthetic passband with an effective width of $800 \AA$ and central wavelength at $4000 \AA$, having steep wings and a flat top (more similar to Sloan than Bessel filters to decrease the errors in the $K$-correction process). Hereafter we refer to it as the $400 \mathrm{~nm}$ band. In a similar way, we defined a second band peaking at $5200 \AA$ with an effective width of $1000 \AA$ and hereafter refer to it as the $520 \mathrm{~nm}$ band. Figure 1 illustrates these synthetic

\footnotetext{
2 This lack of objects known below $z<0.1$ is simply due to their low volumetric rates.
} 


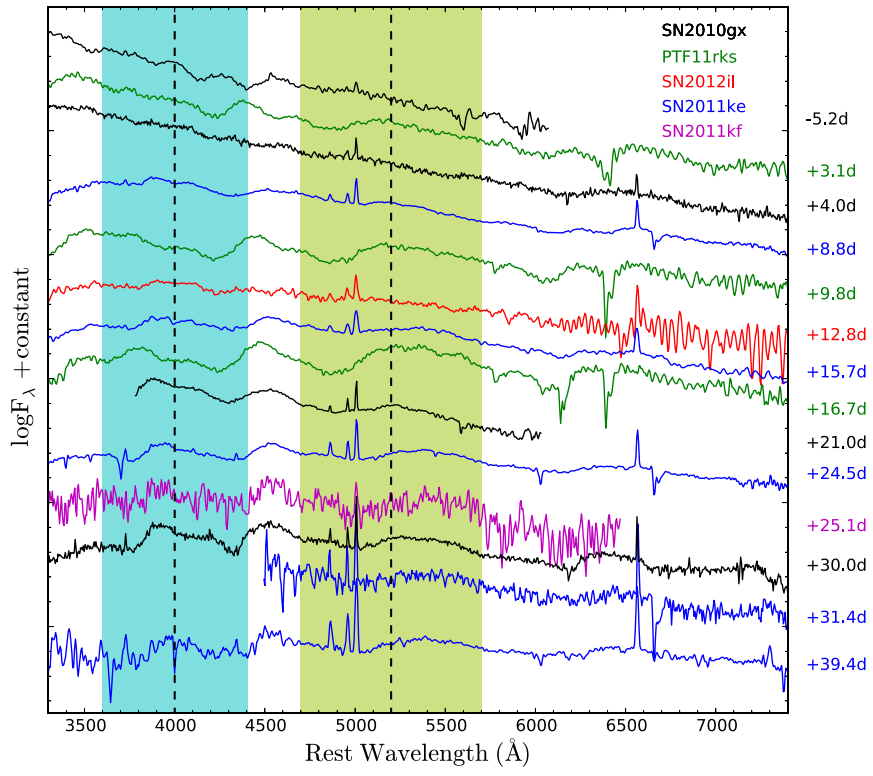

Figure 1. General spectral evolution of SLSN Ic (2005ap-like) from 5 days before maximum light to 40 days after. The phase of each spectrum relative to the light curve peak in the rest frame is shown on the right. These rest-frame spectra have been corrected for Galactic extinction. They have been convolved with a Gaussian function of FWHM $=5 \AA$ and subsequently binned to $5 \AA$ per pixel. The $400 \mathrm{~nm}$ and $520 \mathrm{~nm}$ wave bands are shown in cyan and green, respectively, with their effective centers marked with black dashed lines. The data are from Pastorello et al. (2010a) and Inserra et al. (2013). Table 1 provides a full list of references to the original sources of data for all objects.

(A color version of this figure is available in the online journal.)

passbands and the rest-frame spectral regions lying in them. The choice of these bands was motivated by both the lack of strong spectral features and also by several practical considerations. To maximize the sample size, we required appropriate photometric data that could be $K$ corrected uniformly to these two bands. If we move the primary rest-frame band $(400 \mathrm{~nm}$ band) redder (for example, close to the standard SDSS $r$ band, which is also relatively featureless), we reduce the complete sample from 16 objects to 11 . This is due to a lack of photometric data at the appropriate redshifted wavelength region. Furthermore, we would not then be able to define a second band (for color tests) because a rest-frame SDSS $i$ band (or something similar) would lie beyond the observer's $z$ band in the near-infrared. We lack any substantial data for these objects in the NIR. One could consider going the other way and pushing the primary $400 \mathrm{~nm}$ band bluer, but one must be careful to avoid the strong (and potentially diverse) absorption associated with elements such as Mg II and Si III (see Chomiuk et al. 2011). We did identify a potentially useful region around $3000 \AA$, which is the bluest we could consider given the lack of extensive restframe UV spectroscopy. However, using this would reduce our complete sample to just five objects because of a paucity of data in the observer $u$ or $\mathrm{U}$ bands for objects with $z \lesssim 0.3$.

For each $\mathrm{SN}$ in question, we calculated $K$ corrections from a suitable observer frame filter back to the $400 \mathrm{~nm}$ and $520 \mathrm{~nm}$ bands. The filters chosen were those that had effective central wavelengths most closely matched to $400(1+z)$ and $520(1+z) \mathrm{nm}$, and they are listed in Table 1 . These are typically $g$ and $r$ bands, but the higher redshift objects employ $i$ and $z$ bands as appropriate. For five of the objects, the available photometry only allowed correction to the bluer $400 \mathrm{~nm}$ band. When the observed filters used were Bessel, we converted the

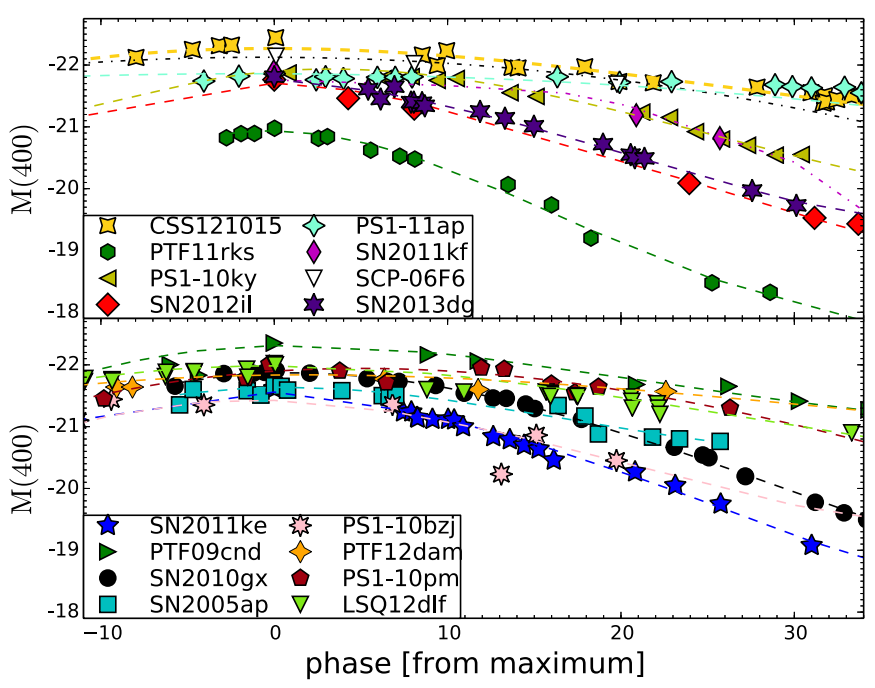

Figure 2. Light curves of our SLSN Ic sample listed in Table 1. The absolute magnitudes, $M(400)$, are for the $400 \mathrm{~nm}$ band after $K$ correction. Time dilation has been applied to report the phase in rest-frame days with respect to maximum light. Polynomial fits to the photometry of the $16 \mathrm{SNe}$ are shown as broken lines. The line fits are all third-order polynomials, apart from SN2011kf and SCP06F6 (second-order polynomials) and CSS121015 (fourth-order polynomial). The objects are split into two panels simply for clarity and illustration purposes.

(A color version of this figure is available in the online journal.)

Vega magnitude into the $\mathrm{AB}$ system using the prescription presented in Blanton \& Roweis (2007).

We computed $K$ corrections with our own python-based code that we call the SuperNova Algorithm for $K$-correction Evaluation (a full description will be published in C. Inserra et al., in preparation along with a code release). We calculated $K$ corrections for each photometric point available in the literature for each SN using an observed spectrum at the same or similar epoch within \pm 4 days from the photometry. For photometric epochs for which no spectra were available, we determined an SED using the multicolor photometric measurements available. If all colors were not available at the specified epoch, we allowed a window of \pm 2 days to select the photometric measurements. If both spectra and photometry were absent, we used our library of spectra that includes 78 spectra from 17 SLSN Ic (including 2007bi-like SLSN). In Table 2 we report the $K$ corrections at key epochs along with the method used to evaluate them (see Appendix B for error treatment and differences between the three methods). A time dilation correction of $(1+z)$ was applied to all epochs with respect to the peak magnitude, which was defined as phase $=0$. The absolute magnitudes were then calculated from

$$
m_{f}=M(400)+\mu+K_{f \rightarrow 400}+A_{f},
$$

where $m_{f}$ is the $\mathrm{AB}$ magnitude in the observed filter $f, \mu$ is the distance modulus calculated from the luminosity distance for our adopted cosmology of $H_{0}=72 \mathrm{~km} \mathrm{~s}^{-1} \mathrm{Mpc}^{-1}, \Omega_{m}=0.27$, and $\Omega_{\Lambda}=0.73, A_{f}$ is the Galactic extinction in the observed filter, and $K_{f \rightarrow 400}$ is the $K$ correction from the observed filter in Table 1 to the synthetic $400 \mathrm{~nm}$ bandpass. The $M(400)$ light curves and the $M(400)-M(520)$ color evolution curves of all of the $\mathrm{SNe}$ after these $K$ corrections are shown in Figures 2 and 3 . The errors on the absolute magnitudes are estimated by propagating the errors of the four terms in Equation (1), including an estimate of the uncertainty in the $K$ correction (details are in Appendix B). 
Table 2

Calculated $K$ Corrections for the Sample of SLSN

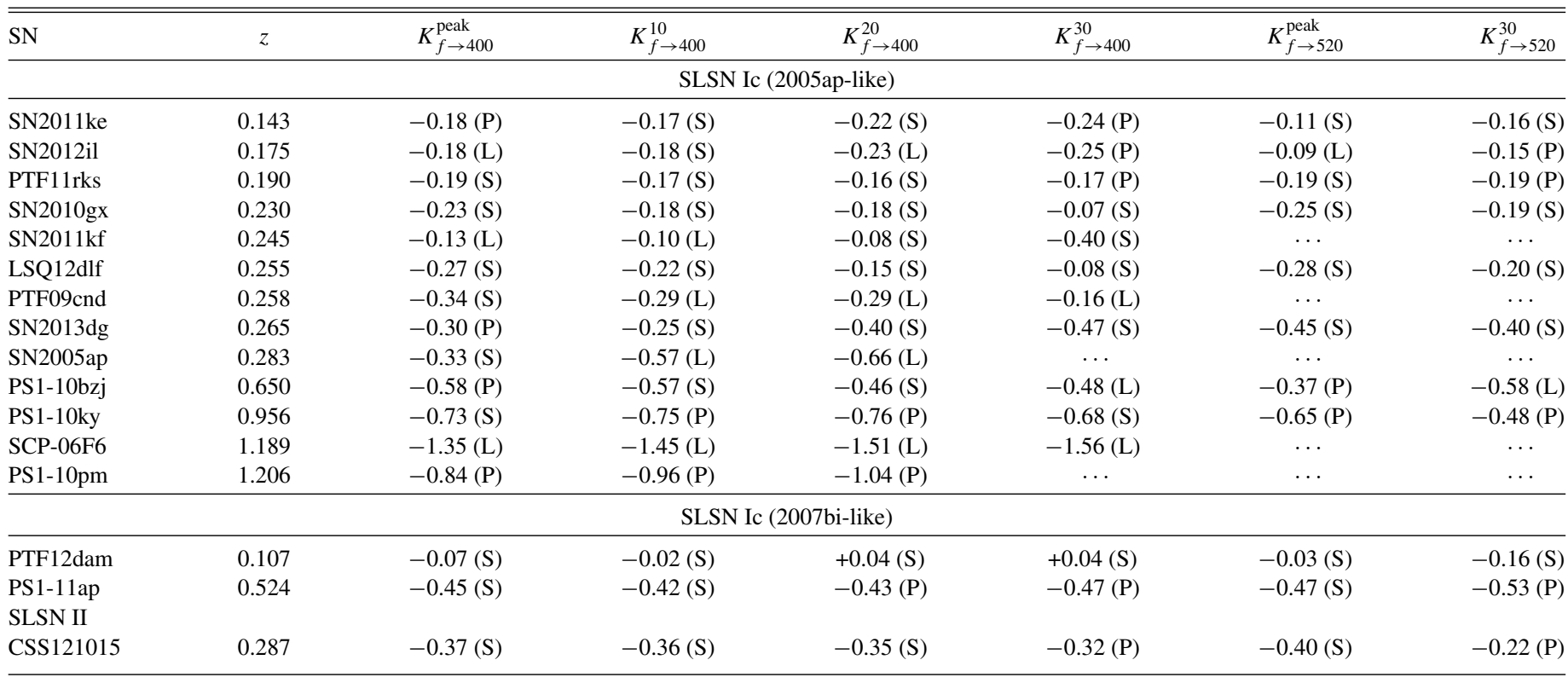

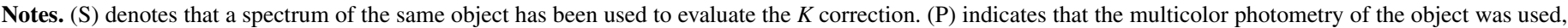
and (L) means that we used our library of spectra.

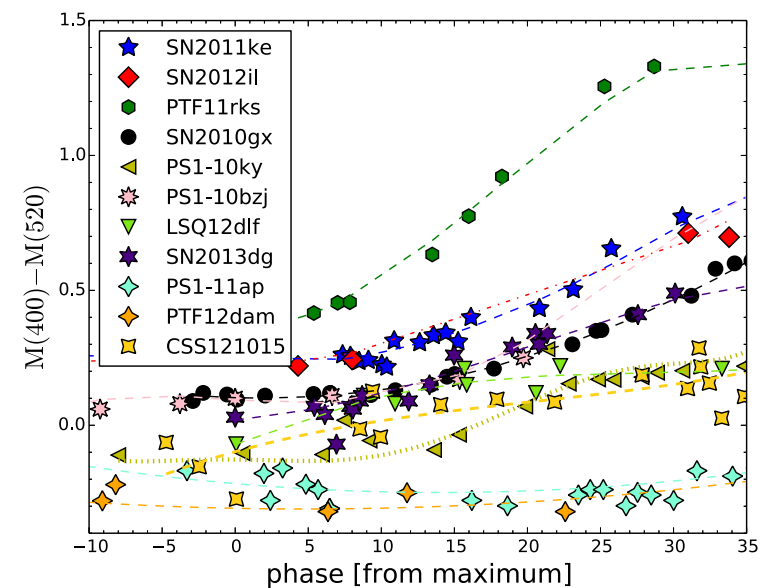

Figure 3. Color evolution as measured from the $M(400)-M(520)$ color index vs. rest-frame phase in days. The reference phase of 0 days is the epoch of maximum in the $400 \mathrm{~nm}$ band; $K$ corrections and time dilation have been applied. Polynomial fits to the color evolution are shown. The color of SN 2012il close to the peak was evaluated using spectra (see Appendix A for details). All line fits represent third-order polynomials (dashed lines), apart from that for PS1-10ky, which was fitted with a fourth-order polynomial (dotted line).

(A color version of this figure is available in the online journal.)

\subsection{Absolute Peak Magnitudes and Light Curve Interpolation}

Most of the sample of $16 \mathrm{SNe}$ had enough data points around the peak to secure a confident estimate of both the value and epoch of the peak magnitude in the $400 \mathrm{~nm}$ band. However, not all SNe had specific measurements exactly at the four key epochs we identified as having useful diagnostic power. These epochs are at peak (defined as 0 days) and after $+10,+20$, and +30 days (rest frame). We employed a consistent method of polynomial fits to the $M(400)$ data points to allow estimates of the magnitudes at these epochs. We found that polynomials of the order of from two to four were sufficient, and the results of these fits are plotted over the data points in Figure 2. Similarly, polynomial fits to the $M(400)-M(520)$ color curves were calculated (either third or fourth order) and are plotted in Figure 3. The specific details for each light curve fit are given in Appendix A. The fits displayed in Figures 2 and 3 and the magnitudes reported in Table 1 are those from the values of the polynomial interpolation at the specific epochs of peak, +10 days, +20 days, and +30 days, unless otherwise noted (see Appendix A for details on each object).

The peak absolute magnitudes of the pure sample (see Section 2) give a mean of $M(400)=-21.81 \pm 0.34$, where the latter number is the standard deviation of the sample. ${ }^{3}$ If the two slowly declining, 2007bi-like SLSN are included, then the extended sample mean and standard deviation are not significantly changed $M(400)=-21.82 \pm 0.32$. We also obtain a similar scatter of $M(400)=-21.86 \pm 0.35$ in the complete case. This raw and uncorrected range is quite encouraging because the bulk of normal (often historically known as "branch" normal) $\mathrm{SNe}$ Ia originally had uncorrected peak magnitudes with scatters of $\pm 0.8,0.6$, and 0.5 in $B V I$, respectively (Phillips 1993). The sample of Prieto et al. (2006) was a factor of 10 larger and showed slightly higher values for the raw standard deviation $\pm 0.9,0.7,0.6$, and 0.6 in $B V R I$, respectively.

In Figure 4, the histograms of the absolute peak magnitudes of our samples are shown. The numbers in each bin are understandably small, but the distribution is consistent with being approximately Gaussian. Considering the statistical errors on the frequency in each bin (see small number confidence limits in Gehrels 1986), we find no evidence for a non-Gaussian distribution of peak magnitudes.

\section{THE PEAK MAGNITUDE DECLINE RATE RELATION}

The now famous Phillips relation for SNe Ia links the rate of decline of a $B$-band SN Ia light curve to the absolute $B$ magnitude at peak (Phillips 1993). The original paper by Phillips brought

\footnotetext{
3 To be clear, we are assuming that these 13 objects are a sample of a larger population; hence the sample standard deviation is calculated using $(n-1)$ as a denominator.
} 

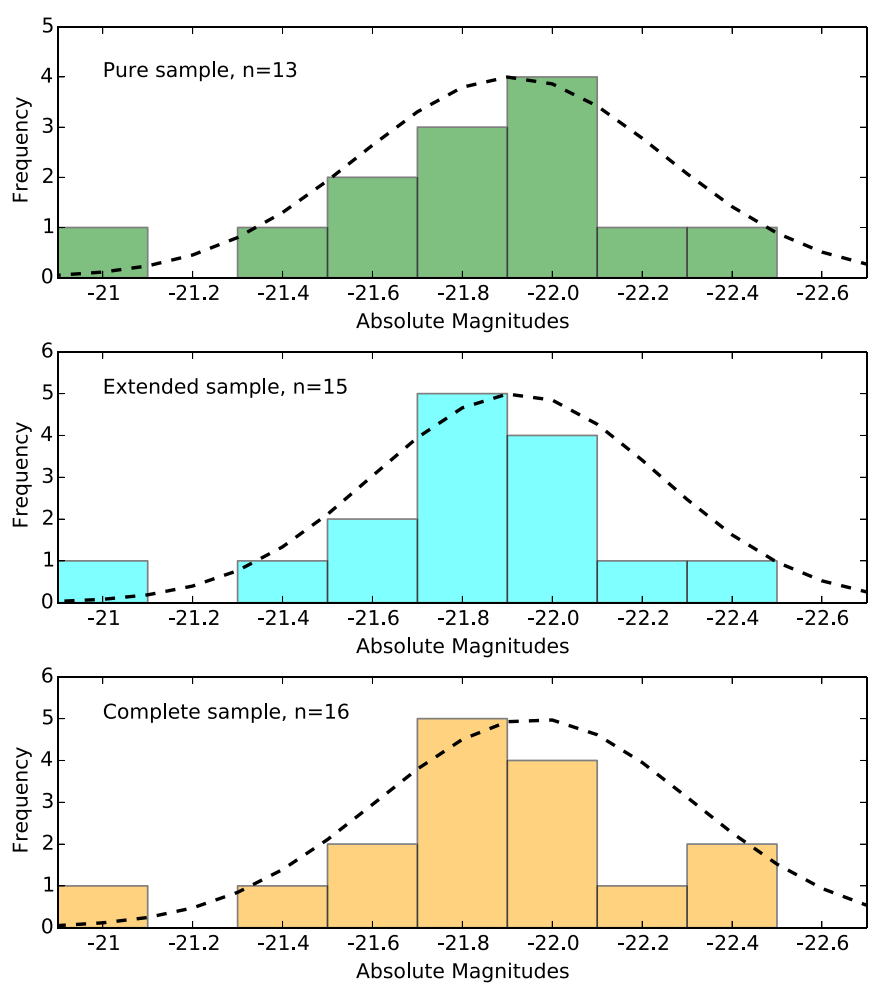

Figure 4. Histograms of the raw (uncorrected) absolute peak magnitudes, $M(400)$, for the three samples defined in Section 2. The dashed lines are leastsquares, best-fit Gaussians. Assuming confidence limits for a small number of events from Gehrels (1986), the Gaussian fits all comfortably fall within the $1 \sigma$ errors of the frequencies.

(A color version of this figure is available in the online journal.)

the intrinsic scatter in SN Ia peak magnitudes down from the raw \pm 0.8 in $B$ to \pm 0.36 , and Prieto et al.'s larger sample produced a scatter of \pm 0.2 . To determine if SLSN are standardizable in a similar way and if the raw scatter of \pm 0.34 mag can be improved upon, we correlated the absolute peak magnitude, $M(400)$, with the amount that the light curve fades from the maximum at three defined periods following peak light.

We chose the timescales of 10,20, and 30 days postmaximum and refer to the difference between peak magnitude at these epochs as $\Delta M_{10}(400), \Delta M_{20}(400)$, and $\Delta M_{30}(400)$, respectively. It is not possible to meaningfully test the extension of the time baseline beyond 30 days because the sample size would reduce considerably due to a lack of data at these epochs. The magnitudes of each $\mathrm{SN}$ at these epochs were estimated from the polynomial fits to the light curve points as described in Section 3.3 with the only exception of the peak magnitude of CSS121015 (see Appendix A). In Figure 5 we plot the relation between $M(400)$ and the decline rates at 10, 20, and 30 days. The plots indicate that the peak magnitude of SLSN Ic does appear to be correlated with decline rate in the same sense as for the Phillips relation for SN Ia. The brighter the peak magnitude (in the rest-frame $400 \mathrm{~nm}$ band), the more slowly they fade. The question then becomes: is this quantitatively useful to reduce the intrinsic scatter in the uncorrected peak magnitudes below the raw scatter of $\pm 0.34 \mathrm{mag}$ ?

The least-squares fits to the data points are shown, and their parameters are given in Table 3 . These suggest that the scatter can indeed be improved upon to between 0.20 and 0.28 , depending on the epoch at which the decline rate is applied and which object sample to include. The most promising relation we find is that for $\Delta M_{20}(400)$, which reduces the scatter from \pm 0.34 mag to \pm 0.20 mag using the pure sample of SLSN Ic. The correlation at 30 days after peak, $\Delta M_{30}(400)$, is essentially the same as that for 20 days. Although the rms scatter is formally larger, the difference is not particularly significant $(0.02$ to 0.04). The Spearman rank-order correlation coefficient for the $\Delta M_{20}(400)$ rate is 0.83 , and the Pearson's $r$ test gives a result of 0.79 and these are also reported in full for each fit in Table 3 .

The Pearson correlation coefficient for the $\Delta M_{20}(400)$ is slightly higher than the $\Delta M_{30}(400)$ and is usually the most commonly employed statistic. It measures the strength of the linear relationship between normally distributed variables. When the variables are not normally scattered or the relationship between the variables is not linear, it may be more appropriate to use the Spearman rank correlation method. Indeed, the Spearman rank correlation method makes no assumptions about the distribution of the data. However, the differences between the coefficients of the fits at 20 and 30 days are fairly small and not obviously significant.

For the extended sample the scatter increases to \pm 0.22 mag ( $\pm 0.26 \mathrm{mag}$ at 30 days), and the Spearman correlation coefficient and Pearson's $r$ are significantly reduced. This simple statistical result and visual inspection of the correlation plots in Figure 5 would suggest that the 2007bi-like, slowly fading SLSN differ from the rest of the SLSN Ic. If they are included in the total sample, then it may result in a distribution with an asymmetric tail and nonnegligible kurtosis. In this case, we should be careful in assuming a normal distribution for the parameters of all SLSN. It is not yet clear if PTF12dam, PS1-11ap, and SN007bi are physically different explosions from the rest of the SLSN Ic, nor is it clear if there is a continuum between the fast and slowly declining events. The next-slowest SLSN Ic are PS1-10pm and SCP-06F6, which do sit comfortably on all of the straight-line fits.

If we would consider the complete sample,then the scatter would increase to $\pm 0.26 \mathrm{mag}$ (or $\pm 0.28 \mathrm{mag}$ at 30 days), with a marginal decrease in the statistical correlation coefficients. We would highlight that we are presenting the results grouped in these three ways simply because the observational characteristics of the three groups are quite easily distinguished in this low to moderate redshift sample. However, if one were working at high redshift, then the subtleties of the different samples and observational classes may be difficult to distinguish.

In Section 3.2 we described how the wavelength window for the synthetic $400 \mathrm{~nm}$ photometric band was chosen. The spectra of all objects in this region are continuum dominated and relatively similar in slope and spectral features. Nevertheless, there are some absorption features present, and the $\mathrm{O}$ II lines are typically the strongest features at $\lambda \geqslant 4200 \AA$. These features are on the red edge of our window and show noticeable absorption before maximum light (Pastorello et al. 2010a; Quimby et al. 2011b; Chomiuk et al. 2011). At peak brightness, the O II absorption at $\sim 4000 \AA$ is relatively shallow and remains weak in the following 20 days (Pastorello et al. 2010a; Inserra et al. 2013). At 30 days past maximum, absorption that is due to $\mathrm{Mg}$ II and $\mathrm{Fe}$ II becomes noticeably stronger than the $\mathrm{O}$ II lines. In our spectroscopic analysis we found two objects, namely PTF11rks and PS1-10bzj, that seem to show noticeable differences in their spectroscopic evolution with respect to the bulk of SLSN Ic. The Mg II and Fe II lines between $3000 \AA$ and $6000 \AA$ and Si II $\lambda 6355$ are stronger in these two objects than the rest of the sample. In the first two weeks after peak, PTF11rks shows absorptionline equivalent widths (EWs) that are a factor of four greater 

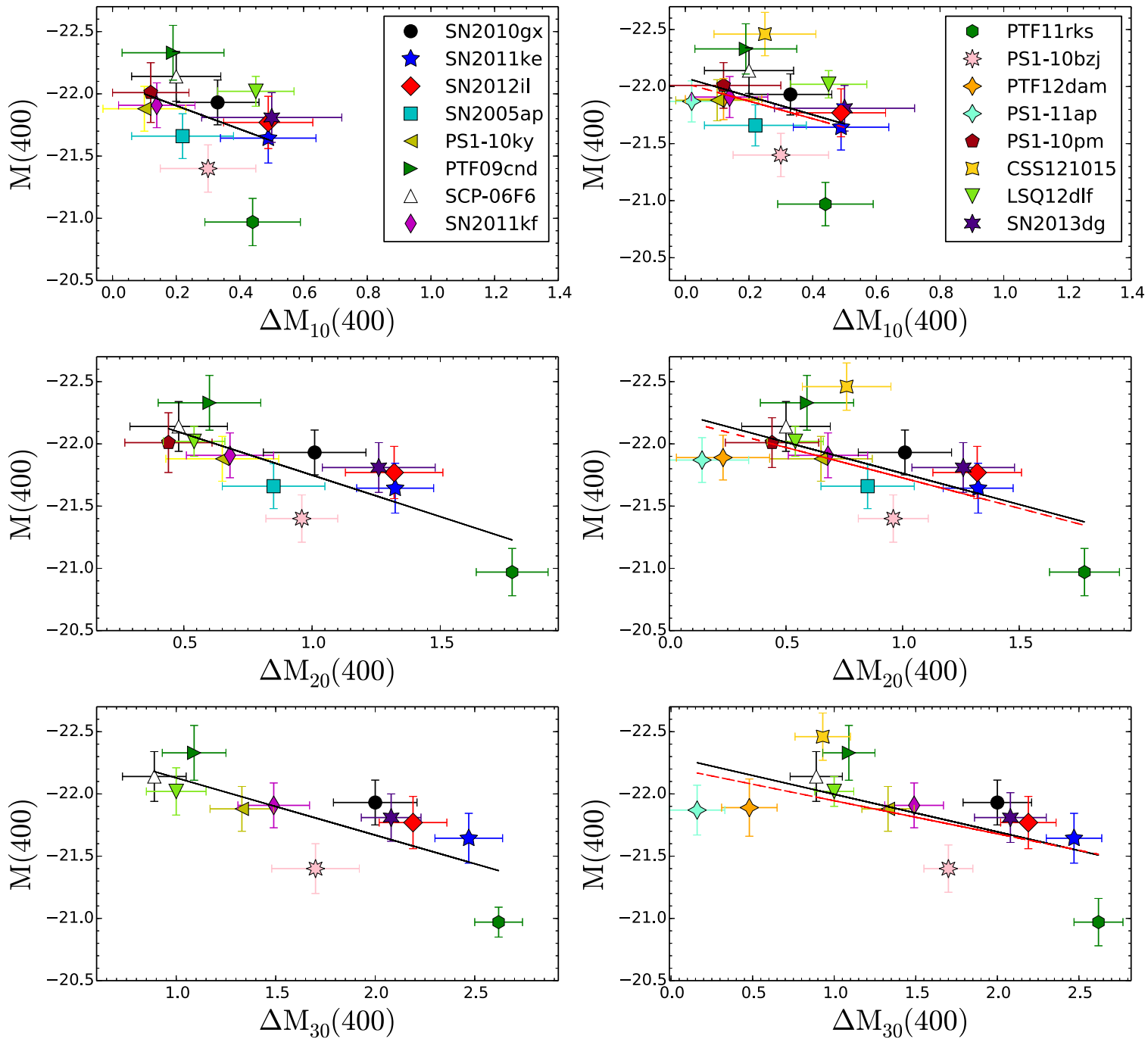

Figure 5. Peak magnitudedecline rate relation. The absolute peak magnitude in the $400 \mathrm{~nm}$ band, $M(400)$, is plotted vs. $\Delta M_{10}(400), \Delta M_{20}(400)$, and $\Delta M_{30}(400)$. The latter three values are measures of the light curve decline (in magnitudes) during the first 10, 20, and 30 days after maximum. The left-hand column of plots shows only the 2005ap-like events (pure sample), and the right-hand column shows all SLSN. The dashed red line is the fit of the extended sample, and the black of the complete sample (see Section 2 for the sample definitions). The SNe magnitudes and the corresponding $\Delta M_{\text {day }}(400)$ are reported in Table 1, and the parameters of the linear regression fits (black line) are listed in Table 3.

(A color version of this figure is available in the online journal.)

than the bulk of the sample $\left(\mathrm{EW}_{\mathrm{PTF} 11 \mathrm{rks}}=4 \pm 1.5 \mathrm{EW}_{\mathrm{SLSNIc}}\right)$. The strengths of these features are noticeable in Figure 1. The PS1-10bzj spectra after maximum light also show a qualitatively similar line intensity evolution, although not as pronounced as PTF11rks. We note that the lower signal-to-noise ratio $(\mathrm{S} / \mathrm{N})$ of the spectra of PS1-10bzj prevent as detailed and quantitative measurements as were possible for PTF11rks, but both appear to evolve on faster timescales than the rest of the SLSN Ic. For completeness, we tested the $\Delta M_{30}(400)$ relation excluding PTF11rks and PS1-10bzj from the pure sample. We found that the scatter was substantially reduced to \pm 0.10 , and we retrieved a Spearman rank-order correlation coefficient of 0.89 , whereas the Pearson's $r$ test is 0.83 . The statistical results are slightly better than those obtained with all objects of the pure sample. This might suggest that these two SNe appear to have spectroscopic and photometric differences compared to the bulk of the population. However, identifying these potential outliers in the distribution is too subtle to be achieved without a well-sampled time series of high signal-to-noise optical and near-UV spectra. Such identification would be difficult for high redshift objects.

\section{THE PEAK MAGNITUDE COLOR EVOLUTION RELATION}

In Section 3.2 we defined two synthetic photometric bands centered on $400 \mathrm{~nm}$ and $520 \mathrm{~nm}$ as regions of the rest-frame spectra that were mostly devoid of strong $\mathrm{SN}$ absorption features and were, on the whole, continuum-dominated. The rest-frame color evolution of the objects, based on these two photometric indices, is plotted in Figure 3. This immediately illustrates a large diversity in color evolution that is visible at the peak and increases substantially during the first 30 days. This is further illustrated in Figure 6, where we plot the absolute peak magnitude $M(400)$ against the color term $M(400)-M(520)$ at peak and after 30 days. The peak absolute magnitude $M(400)$ appears to be color dependent, in the sense that objects that are fainter at peak are redder. If it were a real correlation, one would immediately consider internal host galaxy extinction as a possible explanation, and we discuss this at the end of this section.

A much more striking trend is that the fainter objects tend to become redder faster. In other words, the rate of color change 
Table 3

Fit Parameters and Statistical Results of our Pure, Extended, and Complete Samples

\begin{tabular}{|c|c|c|c|c|c|c|}
\hline Days & $\begin{array}{c}N \\
\text { (objects) }\end{array}$ & $a$ & $b$ & $\begin{array}{c}\sigma \\
(\mathrm{mag})\end{array}$ & Spearman & Pearson \\
\hline \multicolumn{7}{|c|}{$\Delta M_{\text {day }}(400)$ Pure Sample } \\
\hline 10 & 13 & $0.94(0.69)$ & $-22.09(0.21)$ & 0.30 & 0.41 & 0.42 \\
\hline 20 & 13 & $0.67(0.16)$ & $-22.42(0.15)$ & 0.20 & 0.80 & 0.79 \\
\hline 30 & 11 & $0.46(0.15)$ & $-22.59(0.27)$ & 0.23 & 0.83 & 0.75 \\
\hline \multicolumn{7}{|c|}{$\Delta M(400-520)$ pure sample } \\
\hline 30 & 8 & $1.54(0.16)$ & $-22.45(0.08)$ & 0.08 & 0.96 & 0.97 \\
\hline \multicolumn{7}{|c|}{$\Delta M_{\text {day }}(400)$ extended sample } \\
\hline 10 & 15 & $0.77(0.56)$ & $-22.08(0.15)$ & 0.28 & 0.36 & 0.40 \\
\hline 20 & 15 & $0.49(0.14)$ & $-22.21(0.12)$ & 0.22 & 0.65 & 0.70 \\
\hline 30 & 13 & $0.26(0.11)$ & $-22.21(0.17)$ & 0.26 & 0.64 & 0.60 \\
\hline \multicolumn{7}{|c|}{$\Delta M(400-520)$ extended sample } \\
\hline 30 & 10 & $1.10(0.23)$ & $-22.19(0.10)$ & 0.13 & 0.83 & 0.88 \\
\hline \multicolumn{7}{|c|}{$\Delta M_{\text {day }}(400)$ complete sample } \\
\hline 10 & 16 & $0.81(0.62)$ & $-22.08(0.17)$ & 0.31 & 0.33 & 0.37 \\
\hline 20 & 16 & $0.50(0.17)$ & $-22.26(0.14)$ & 0.26 & 0.59 & 0.63 \\
\hline 30 & 14 & $0.30(0.12)$ & $-22.30(0.17)$ & 0.28 & 0.61 & 0.64 \\
\hline \multicolumn{7}{|c|}{$\Delta M(400-520)$ complete sample } \\
\hline 30 & 11 & $1.27(0.27)$ & $-22.30(0.11)$ & 0.19 & 0.80 & 0.85 \\
\hline
\end{tabular}

Notes. Least-squares fits for an unweighted linear fit of the form $M_{\max }(400)=a \Delta M_{\mathrm{day}}(400)+b$ with uncertainties in parentheses. The $\sigma$ is the standard deviation of this fit. The last column gives the Spearman rank-order correlation coefficient and the Pearson correlation coefficient $r$. Because the errors on each value of $M(400)$ are fairly similar, a weighted calculation is not significantly different. For completeness, we have reported the fit values obtained excluding CSS121015, but SN2005ap and PS1-10 pm are left out of the 30 day decline calculations because they do not have enough data.

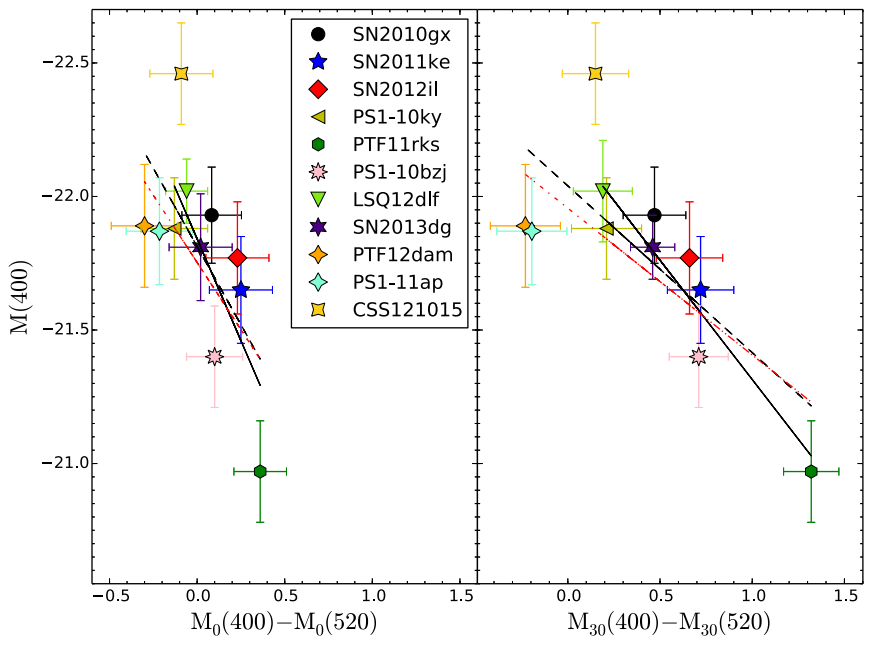

Figure 6. Peak magnitudecolor evolution relation. This plot illustrates the apparent dependence of peak magnitude on the color and color-evolution rate. The left panel plots the $M(400)$ peak magnitude vs. the color at peak brightness. The right panel plots the same $M(400)$ at peak vs. the color at +30 days (rest frame). The black line fit refers only to the pure sample (2005ap-like objects), the red dot-dashed line fits the extended sample (which includes the 2007bi-like objects), and the black dashed line fits the complete sample.

(A color version of this figure is available in the online journal.)

appears to be correlated with peak magnitude. This strong dependency is displayed in Figure 7, where we show that the peak absolute magnitude is correlated quite tightly with the rate of color evolution. In the pure sample, we are reduced to only eight objects because of the requirement for data covering the rest-frame $400 \mathrm{~nm}$ and $520 \mathrm{~nm}$ bands at both peak and at +30 days. This could potentially bias the result toward a positive result and artificially reduce the scatter around the straight-line fit. However, the rms scatter around the linear least-squares fit is reduced to only 0.08 mag for the eight objects of the pure sample. The two statistical correlation tests result in 0.96 for the Spearman coefficient and 0.97 for the $r$ Pearson's coefficient. For the 10 objects of the extended sample, the residual rms is still quite low at $0.13 \mathrm{mag}$. It increases to $0.19 \mathrm{mag}$ for the complete sample when CSS121015 is included. From Figures 6 and 7 it is clear that PTF12dam and PS1-11ap are systematically bluer than the rest of the SLSN Ic, both at peak and at +30 days, and CSS121015 also sits significantly above the best linear fit.

For completeness, we also checked the correlation over the 20 day evolution timescale and found a poorer correlation. For $\Delta M(400-520)_{20}$ we found an rms of $0.19 \mathrm{mag}$ (rising to 0.27 when CSS121015 is included) and lower statistical coefficients than for the $\Delta M(400-520)_{30}(0.38$ for the Spearman coefficient and 0.80 for the $r$ Pearson's coefficient). Hence the 30 day color rate evolution seems significantly better. Although the numbers are low, this peak magnitudecolor evolution relation appears to be quite promising as a method of standardizing the magnitudes of SLSN. The rms scatter is surprisingly low, even with the inclusion of the most outlying three objects in the complete sample. The very tight correlation for the eight SLSN Ic of the pure sample makes it imperative that this relationship be tested with a larger sample.

The major uncertainty is extinction within the host galaxies. We have made no correction for internal dust extinction, and it is difficult to quantitatively measure reddening from either the colors, the spectrum slope, or the interstellar medium (ISM) lines in this small sample (indeed we do not detect ISM Na I lines from the ISM in the host galaxies for any SLSN). One might be concerned that the left panel in Figure 7 was 

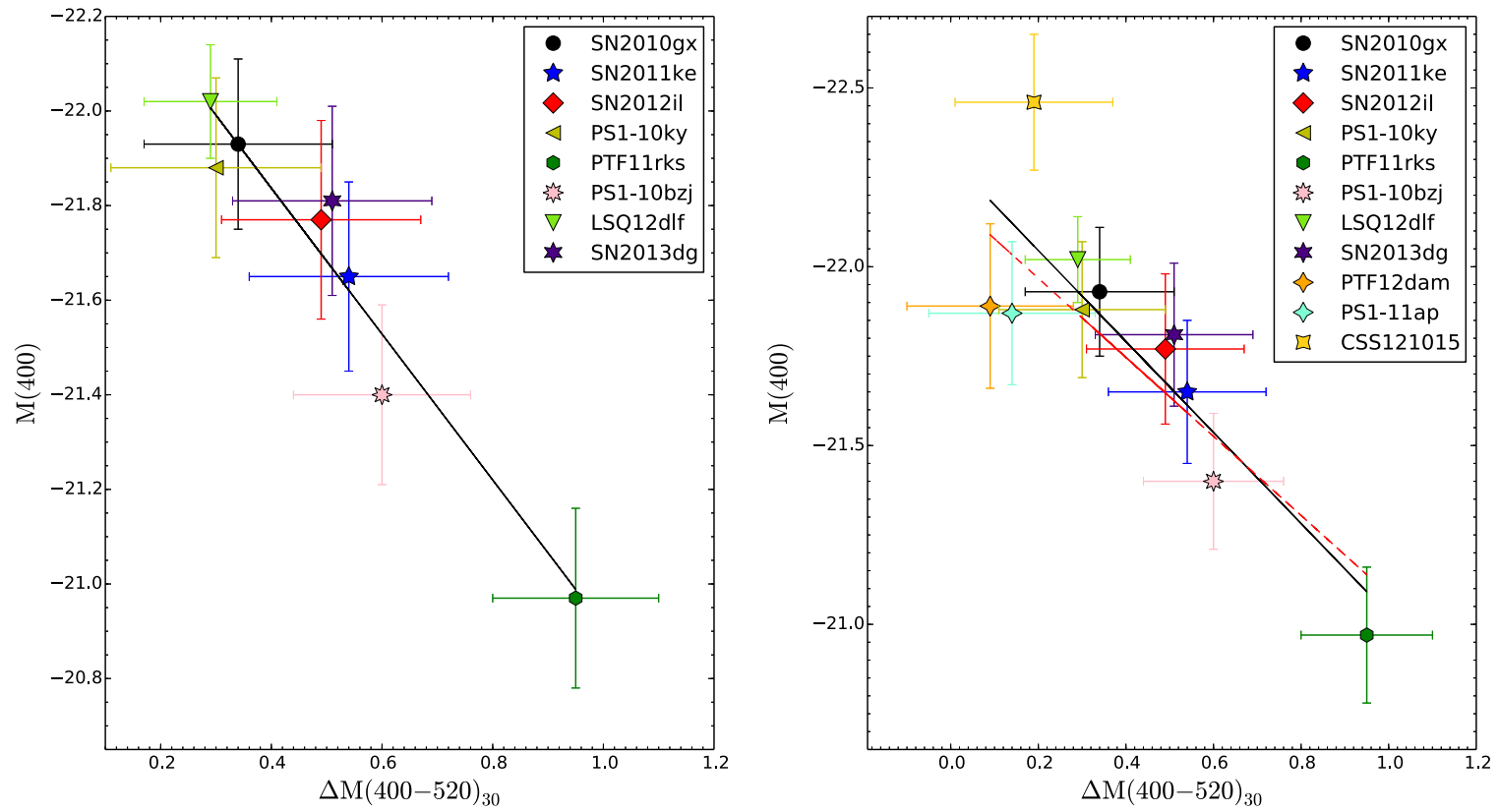

Figure 7. Peak magnitudecolor evolution relation. Absolute peak magnitudes in the $400 \mathrm{~nm}$ band, $M(400)$, vs. the color change from peak to +30 days (rest frame). We define $\Delta M(400-520)_{30}=\left(M_{0}(400)-M_{0}(520)\right)-\left(M_{30}(400)-M_{30}(520)\right)$. The bands at $400 \mathrm{~nm}$ and $520 \mathrm{~nm}$ are defined in Section 3.2, and the values for $\Delta M(400-520)_{30}$ are reported in Table 1. Left: a plot of eight of the pure sample that have the necessary bands covered in the observer frame to calculate this color term. Right: 11 objects of the complete sample that have the necessary data. The black lines are least-squares straight-line fits to all data in each plot. The red dotted line leaves out CSS121015. The parameters of these line fits are listed in Table 3.

(A color version of this figure is available in the online journal.)

a reddening effect if we simply observed that the fainter SLSN were redder at peak. An argument against it being extinction is that both PTF11rks and PS1-10bzj have a qualitatively different evolution in their spectral features (deeper Mg II, Fe II, and Si II absorption) than the rest of the pure sample. This suggests cooler photospheric temperatures rather than an extinction effect (see discussion in Section 4). The brightest object (CSS121015) is also spectroscopically different in that $\mathrm{H} \alpha$ was detected, and it may well be physically different from the others. Constant extinction values might explain the connection between color and luminosity, but they cannot easily account for the observed color evolution in the light curves. The distinctive time evolution of the $M(400)-M(520)$ color could, in principle, be due to time-variable internal dust extinction. However, to our knowledge, such time variability has not been observed before toward any SN. Time-variable ISM and circumstellar medium absorption lines have been observed toward SN Ia (Patat et al. 2007), but these have not been linked with variable extinction toward the SN photosphere. In the future, a more sophisticated approach would be to combine fits for decline and color evolution into one standardization procedure as has been successfully done for SN Ia, e.g., methods like SALT2 (Guy et al. 2007), or to use Bayesian methods to simultaneously model dust and color effects as in Mandel et al. (2009, 2011). Additionally, we plan to look for extinction signatures by searching for objects with identical photospheric spectra but which have different colors and continuum slopes. This would ideally include near-infrared photometric fluxes to anchor the SED fits securely. Both of these approaches require a more extensive data set than we have presented here, which should be possible given the ongoing low-redshift surveys such as PESSTO and La Silla QUEST (Baltay et al. 2013; S. J. Smartt et al., in preparation), CRTS (Drake et al. 2009) and iPTF (Cao et al. 2013).

\subsection{A Control Test: Application to Type Ib/c}

As a sanity check we investigated whether the relations we appear to have discovered for SLSN Ic also manifest themselves in the normal type $\mathrm{Ib} / \mathrm{c} \mathrm{SN}$ population and hence whether we are recovering characteristics that are commonly displayed in more normal hydrogen-poor stellar explosions. We performed the same analysis with a sample of well-observed strippedenvelope SN (type Ic, Ib, IIb), with the only difference from the SLSN Ic analysis being the choice of the reference bands. We chose the Johnson $B$ band with central peak wavelength at $4448 \AA$ and Johnson $V$ band $5505 \AA$ because of the excellent sampling of the low-redshift observed $\mathrm{SN} \mathrm{Ib/c}$ in these standard wave bands (which were not in need of any $K$ correction). As shown in Figure 8, there is no relation between the peak magnitudes and the decline rates at 10,20 , and 30 days or with the color evolution. As expected, we find a large scatter of peak magnitudes and no obvious correlations, which mimic the findings for the SLSN. The mean and raw scatter in the $M_{B}$ values for type $\mathrm{Ibc} \mathrm{SNe}$ are $-17.42 \pm 0.90$, and this is not improved when any linear fits are applied.

\section{HUBBLE DIAGRAM AND RESIDUALS}

If we assume that SLSN Ic can be used as standardizable candles and then apply the $\Delta M_{30}$ decline relation (calibrated with 11 objects) and the peak magnitudecolor evolution relation (with the 8 objects reported in Section 5), then we can use these as a preliminary test to check whether they fall in a reasonable region of the Hubble diagram, as shown in Figure 9. The small number of objects and lack of a low-redshift anchor prevents any detailed discrimination between cosmology models, but consistency checks of the relative distances for a cosmology with $\Omega_{\Lambda}=0$ are possible. 

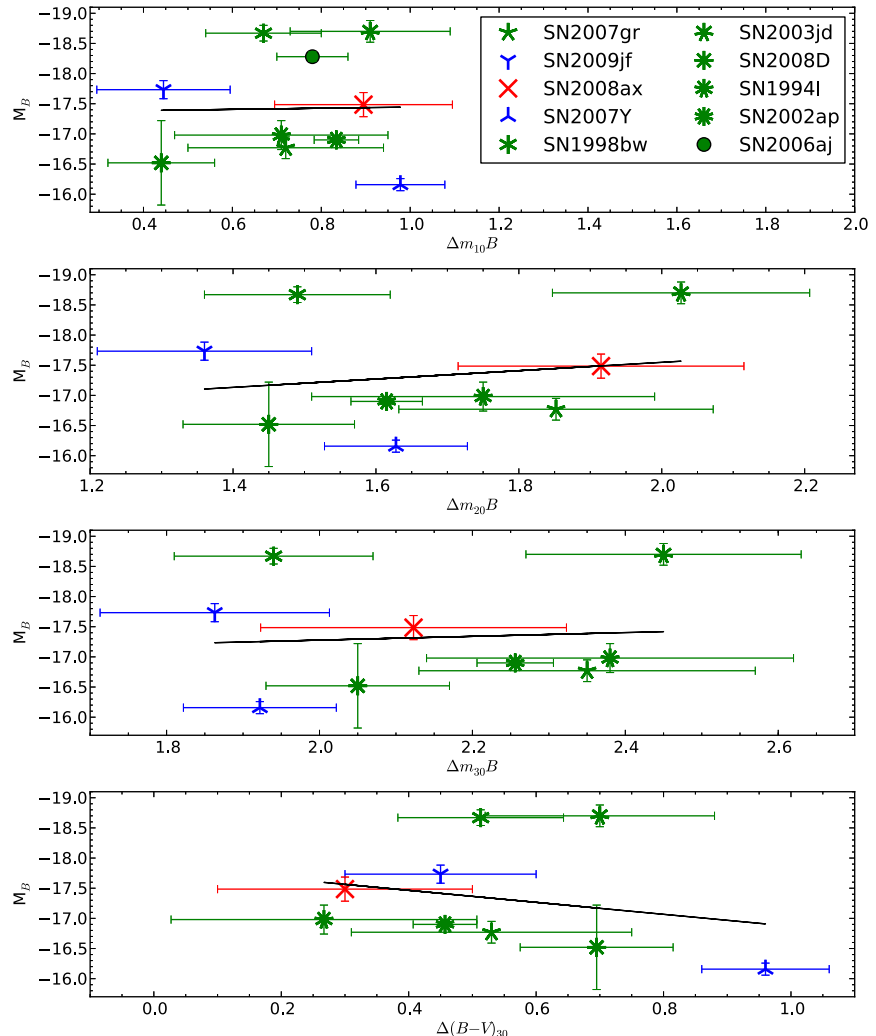

Figure 8. Peak magnitudedecline relation and the peak magnitudecolor evolution relation for 10 stripped-envelope CCSN. Absolute magnitudes in the $B$ band are plotted vs. $\Delta m_{10}(B), \Delta m_{20}(B)$, and $\Delta m_{30}(B)$, analogous to the SLSN sample in Figure 5. The $\Delta(B-V)_{30}$ measures the color evolution in $B-V$ over 30 days. The data sources are as follows: the type Ic SN 2007gr (Valenti et al. 2008b; Hunter et al. 2009), 1998bw (Galama et al. 1998; McKenzie \& Schaefer 1999; Sollerman et al. 2000; Patat et al. 2001), 2003jd (Valenti et al. 2008a), 2008D (Mazzali et al. 2008; Soderberg et al. 2008; Modjaz et al. 2009), 1994I (Richmond et al. 1996), 2002ap (Pandey et al. 2003; Foley et al. 2003; Yoshii et al. 2003; Tomita et al. 2006), and 2006aj (Campana et al. 2006; Cobb et al. 2006; Mirabal et al. 2006; Pian et al. 2006; Sollerman et al. 2006); the type Ib SN 2007Y (Stritzinger et al. 2009), 2009jf (Valenti et al. 2011), and the type IIb SN 2008ax (Pastorello et al. 2008).

(A color version of this figure is available in the online journal.)

We evaluated the distance moduli with the simple formalism $\mu=m-M+\alpha \Delta M$, where $\Delta M$ refers to the standardization relation used and $\alpha$ is a free parameter. This parameter $\alpha$ is allowed to vary and to minimize the $\chi^{2}$ between the fit to the distance moduli and those for different cosmologies. Because of our statistically limited data set, we can only determine if the data are consistent with a $H_{0}=72, \Omega_{M}=0.27, \Omega_{\Lambda}=0.73$ universe. We found a satisfactory fit and the lowest $\chi^{2}$ for this concordant cosmology model. If we attempt a fit with $\Omega_{\Lambda}=0$, $\Omega_{M}=1$, then we get a $\chi^{2}$ that is a factor of 10 worse and an unsatisfactory Hubble diagram fit. This simply illustrates that the relative distances to these SLSN are not compatible with a cosmological model with $\Omega_{\Lambda}=0$, but we do not have enough statistics to test it any further.

The results of the best fit are displayed in Figure 9. It also indicates that distance indicators in the range of $0.6 \lesssim z \lesssim 3$, where SLSN Ic are already discoverable with the current generation of telescopes, may give us some leverage on $\Omega_{M}$ because the difference between $\Omega_{M}=0.20$ and 0.32 is $\sim 0.3 \mathrm{mag}$, which would be well within the reach of a sample of 1020 SLSN Ic in the redshift range $z=2-3$. The SLSN will probably not achieve a competitive precision to rival $\Omega_{\lambda}$ measurements from either baryon acoustic oscillations (Eisenstein et al. 2005;
Percival et al. 2010) or cosmic microwave background experiments (Dunkley et al. 2009; Komatsu et al. 2011), as already noted by Suzuki et al. (2012). However, because SLSN are the only current alternative to SN Ia for estimating radial distances at high redshift, it is worth exploring whether they can constrain the time-varying nature of $w(z)$, the dark energy equation-ofstate parameter. The study of SLSN at high $z$ is also a promising avenue to explore the nature of the explosions and sources of luminosity and to investigate if there are changes in their properties with epoch and environment (see Appendix C).

In the future, we plan to tie down the calibrations for these potential standardizable candles in the lower redshift regime of $0.1<z<0.3$ simply by increasing the number of objects discovered and monitored in detail. Their cosmological usefulness may stem primarily from their ability to be discovered and studied in the redshift regime beyond that for SN Ia, which is currently around $z=1.55$ (Rodney et al. 2012).

\section{FUTURE USE OF SLSN AS HIGH-REDSHIFT DISTANCE PROBES}

If our results are confirmed with a larger sample, then these SLSN show potential as new cosmological probes stretching well into the epoch of deceleration. At $z \geqslant 1$, the synthetic passbands at 400 and $520 \mathrm{~nm}$ move into the near-infrared. We primarily chose the central wavelength and width of these restframe bands because of the lack of strong features in the SLSN Ic spectra (see Section 3.2), but we also considered the location and width of the redshifted passbands in the observer frame at $1 \lesssim z \lesssim 4$. In Figure 10 we show the two bands redshifted at $z=1,2,3,4$. The $400 \mathrm{~nm}$ band covers a wavelength region similar to the Sloan $i$ band, the NIR $J$ band, the $H$ band, and partially the $K$ band, respectively. Observations in these bands would mitigate the dependence of the cross-filter $K$ correction on the spectral template used. Similarly, the $520 \mathrm{~nm}$ band would be reasonably well covered at $z=1,2,3$ by the standard filter bands $Y, H$, and $K$, respectively. No standard ground-based filter band is found to match the $520 \mathrm{~nm}$ band at $z=4$ because of the atmospheric absorption from $\sim 2.5 \mu \mathrm{m}$ to $\sim 3.5 \mu \mathrm{m}$. As an example, typical average magnitudes of SLSN in the rest-frame $400 \mathrm{~nm}$ band would correspond to $J \sim 23.0, H \sim 23.8$, and $K \sim 24.3$ mag at $z=2,3,4$, respectively. The exposure times with the Very Large Telescope and HAWK-I are between 2 and $4 \mathrm{hr}$ to reach a signal-to-noise of 10 for these magnitudes. Although the Wide Field Camera 3 (WFC3) on the Hubble Space Telescope can only get to $J$ and $H$ equivalent wavelengths (F110W and F160W), one can reach improved photometric precision $(\mathrm{S} / \mathrm{N} \simeq 50)$ in manageable exposure times of between $300 \mathrm{~s}$ and $3000 \mathrm{~s}$ on source. Although these are feasible as dedicated follow-up projects, neither HAWK-I nor WFC3 are appropriate survey instruments to find them in large numbers because of their limited field size (tens of square arcminutes). A more appropriate search engine is a planned survey such as SUDSS (Survey Using Decam for Superluminous Supernovae) using the Dark Energy Camera on the CTIO Blanco $4 \mathrm{~m}$ telescope. This project has a goal of discovering 200 SLSN out to $z \simeq 4$ over three years by imaging approximately $24 \mathrm{deg}^{2}$ (in griz) to $i \simeq 25$ every 14 days. The planned Subaru/Hyper Suprime-Cam deep survey will also target these high redshift objects, with encouraging numbers estimated by Tanaka et al. (2012). The NIR imaging follow-up would be required to reach the rest-frame $400 \mathrm{~nm}$ and $520 \mathrm{~nm}$ bands and exploit our proposed standardizing of the peak magnitudes. Identification of the redshifts would not require spectra in the NIR but could be 

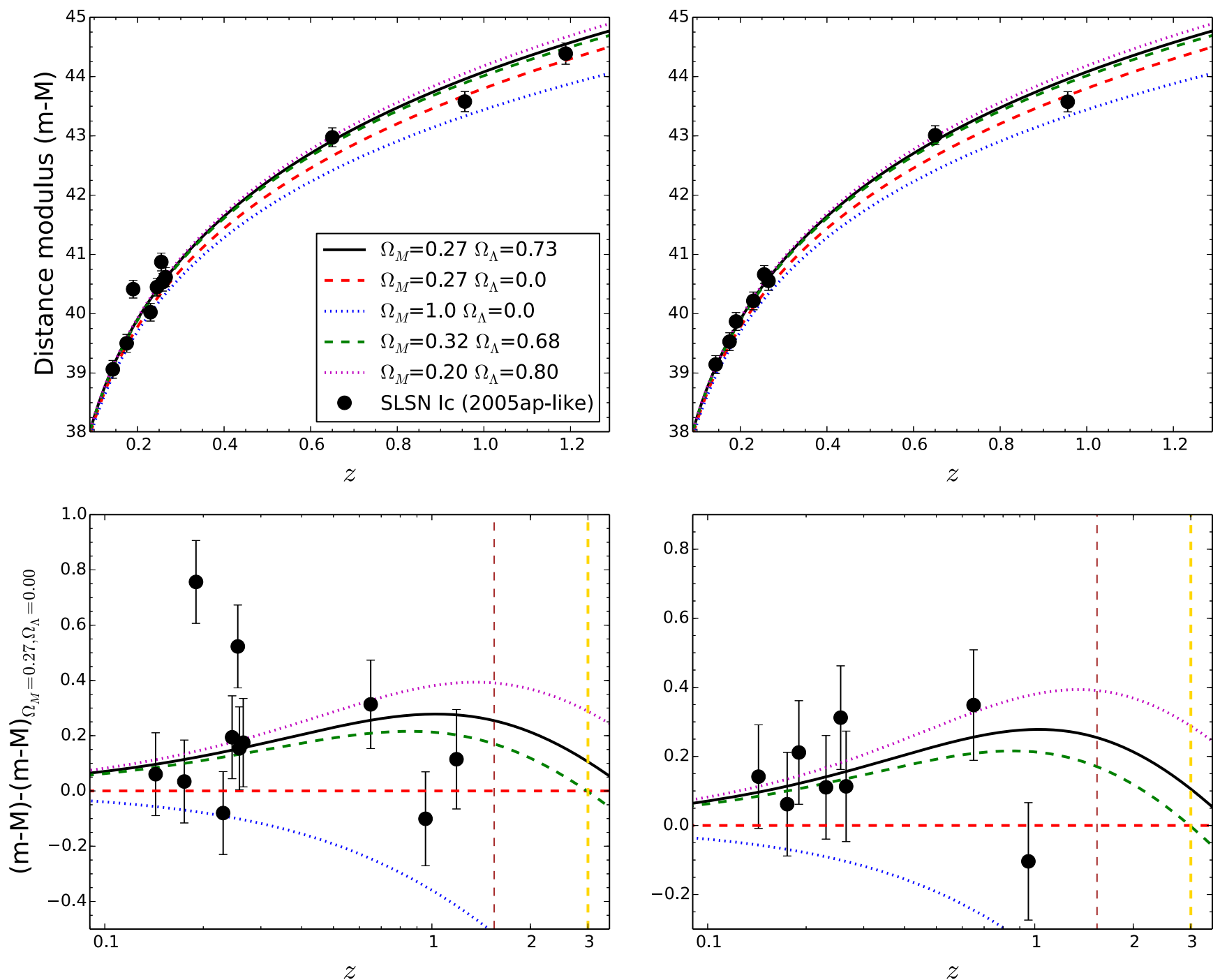

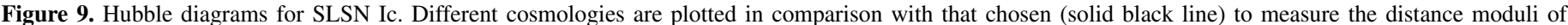

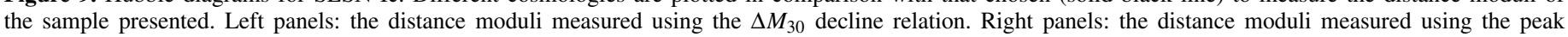

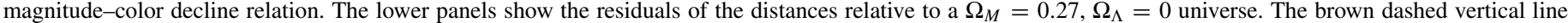

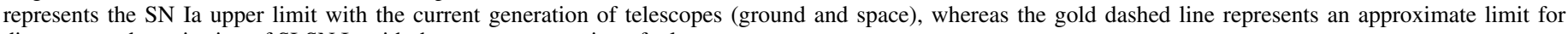
discovery and monitoring of SLSN Ic with the current generation of telescopes.

(A color version of this figure is available in the online journal.)

done in the optical observer frame (rest-frame UV wavelengths between 1200 and $2500 \AA$ ) where the SLSN are predicted to peak around $i=23.5$ at $z \simeq 3$.

Despite their enormous luminosity and potential for standardizing the peak magnitudes, two practical difficulties remain. The first is that we have only 8 to 13 SLSN (depending on sample selection) that have sufficient multicolor data at the epochs and filters required, so increasing the sample is mandatory. The second is their low volumetric rate of cosmic production. A rough estimate of their rate in the local universe was provided by Quimby et al. (2013b), who reported a rate of $32_{-26}^{+77}$ events $\mathrm{h}_{71}^{3} \mathrm{Gpc}^{-3} \mathrm{yr}^{-1}$ at a weighted redshift $\bar{z}=0.17$, although this was based on only one detected object (SN2005ap) in the TSS. Although the number is uncertain, the TSS and PTF (Quimby et al. 2011b) do demonstrate that the SLSN rate in the local universe is low compared to that of SN Ia $(0.01 \%)$. McCrum et al. (2014b) has estimated a rate of $6_{-2.4}^{+3.6} \times 10^{-5}$ and $1_{-0.8}^{+2.3} \times 10^{-4}$ of the CCSN rate within $0.3 \leqslant z \leqslant 1.4$. However, at redshifts $z \geqslant 1.5$, Cooke et al. (2012) suggested that the rate could be significantly higher, possibly as a consequence of decreasing metallicity and increasing cosmic star-formation rate density. The SLSN Ic may require low metallicity to be produced (e.g., Chen et al. 2013; Lunnan et al. 2014), but the efficiency of producing an SLSN from progenitor systems is still unknown, and the progenitor stellar mass range is undetermined. Future surveys exploring the low-, medium-, and high-redshift distances are necessary to strengthen or falsify the findings here reported and hence make use of SLSN as high-redshift distance probes.

Looking further ahead, detecting SLSN Ic out to redshifts of $z \simeq 10$ is quite plausible but requires space-based surveys to sample the rest-frame $400 \mathrm{~nm}$ and $520 \mathrm{~nm}$ bands proposed here. As an illustration of what would be required, we plot the apparent $\mathrm{AB}$ magnitudes of SLSN Ic between redshifts 1 and 10 in Figure 11. These are the redshifted $M(400)$ magnitudes in the $\mathrm{AB}$ system, from the conventional definition

$$
m_{\mathrm{AB}}=M(400)+5 \log \left(D_{\mathrm{L}}(z) / 10 \mathrm{pc}\right)-2.5 \log (1+z),
$$

where $M_{\mathrm{AB}}$ is the apparent $\mathrm{AB}$ magnitude at the redshifted wavelength, and $D_{\mathrm{L}}$ is the luminosity distance with our chosen cosmology. We label the wavelengths and filter systems required 

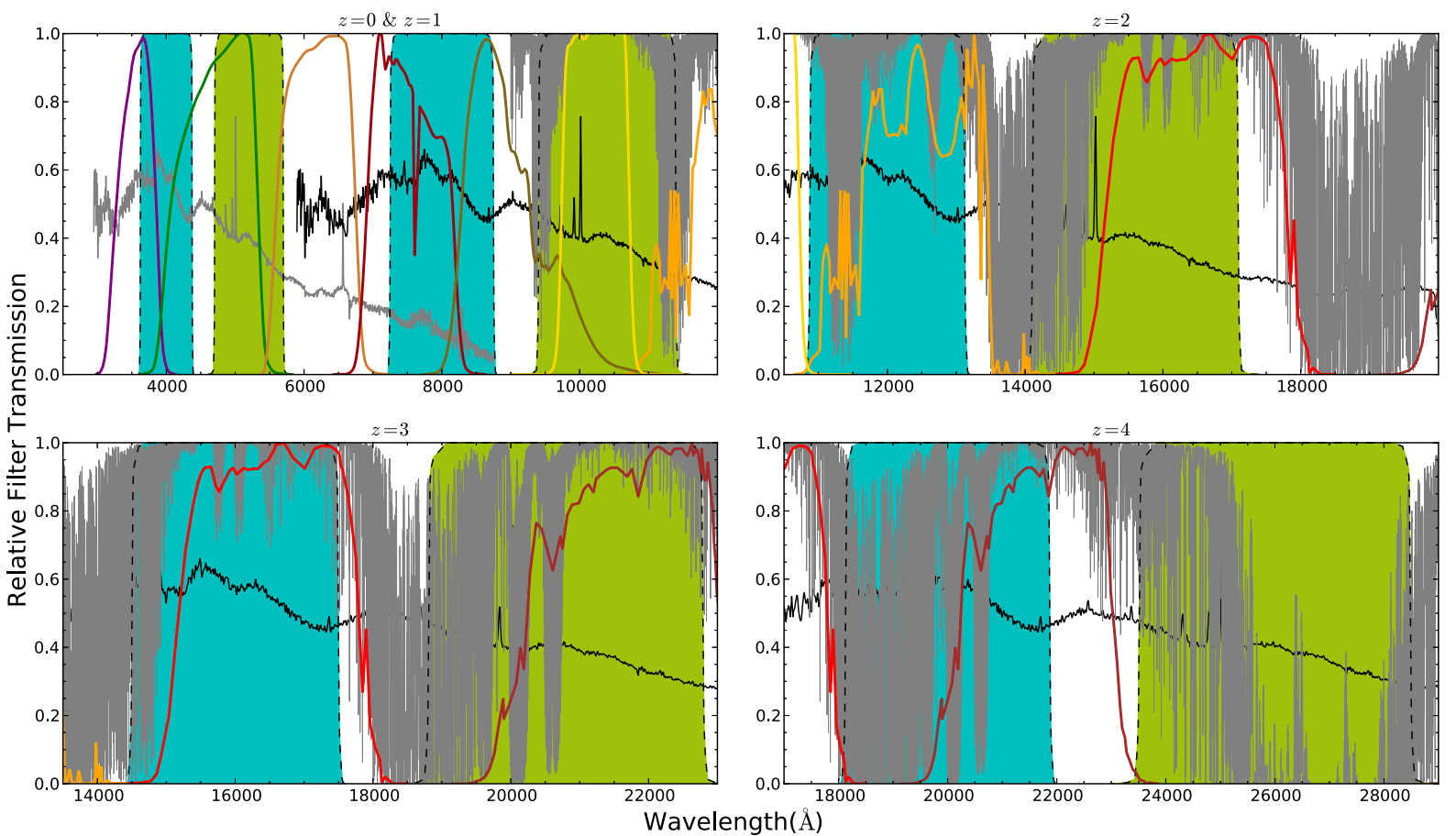

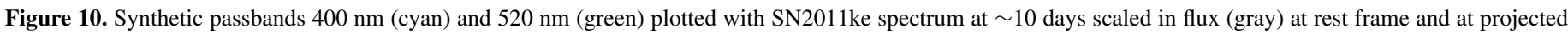

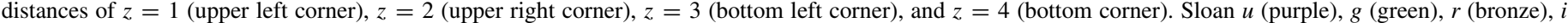

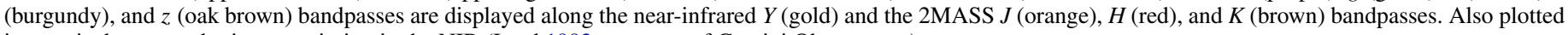
in gray is the atmospheric transmission in the NIR (Lord 1992; courtesy of Gemini Observatory).

(A color version of this figure is available in the online journal.)

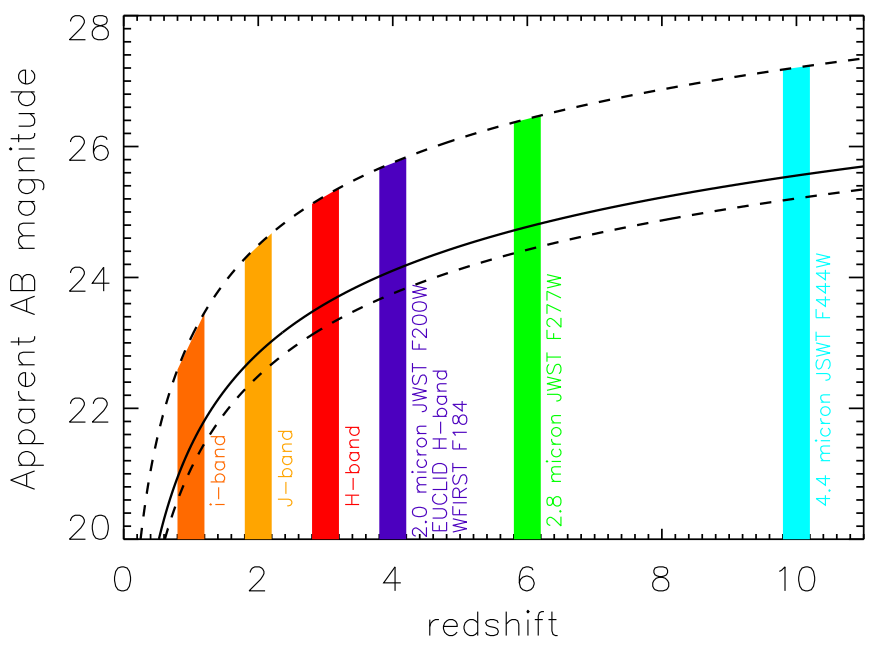

Figure 11. At higher redshift the only possibility of observing the $400 \mathrm{~nm}$ and $520 \mathrm{~nm}$ bands is using future space-based telescopes. This figure illustrates the predicted $\mathrm{AB}$ magnitude for SLSN Ic out to $z \simeq 10$. The solid line is the peak magnitude of $M(400)=-21.86$ derived here. The lower dotted line is a $1 \sigma$ scatter on the peak magnitudes, and the upper dotted line illustrates the depth required to reach +20 days after peak to apply the $\Delta M_{20}(400)$ correction discussed here (addition of the typical 1. 3 decline). No foreground extinction has been applied to the estimated magnitudes because the Milky Way foreground will be minimal at these wavelengths.

(A color version of this figure is available in the online journal.)

to cover the rest-frame $400 \mathrm{~nm}$ band at specific wavelengths. The magnitudes are well within the capabilities of the future EUCLID $^{4}$ (Laureijs et al. 2011), WFIRST ${ }^{5}$, and James Webb Space Telescope (JWST; specifically NIRCam ${ }^{6}$ ) missions, even

\footnotetext{
4 http://www.euclid-ec.org

5 http://wfirst.gsfc.nasa.gov/

6 http://www.stsci.edu/jwst/instruments/nircam/
}

out to redshifts of $z \simeq 10$. However, the luminosity distances at these redshifts are exactly what we would like to probe using our standardization for the low-redshift objects. A major uncertainty will be the rates and the numbers that these space missions may discover in their general survey and custom-designed survey modes (see Tanaka et al. 2012; Cooke et al. 2012 for initial estimations of detection rates of high-redshift $\mathrm{SNe}$ ).

\section{CONCLUSION}

From a study of 16 well-observed and well-sampled SLSN Ic in the redshift range $0.1<z<1.2$, we have investigated their use as standard and standardizable candles. We defined two synthetic photometric wave bands and applied quantitative $K$ corrections from observed spectra to compare their magnitudes in consistent rest-frame wave bands. These two wave bands are centered on $400 \mathrm{~nm}$ (with a width of $80 \mathrm{~nm}$ ) and $520 \mathrm{~nm}$ (with a width of $100 \mathrm{~nm}$ ) and were chosen because the spectra are continuum dominated and relatively free from absorption features. The raw scatter of the uncorrected $M(400)$ absolute peak magnitudes is $\pm 0.35 \mathrm{mag}$, which is immediately of interest for cosmological use, given that they have a mean peak magnitude of $M(400)=-21.86 \mathrm{mag}$, around 2 mag brighter than SN Ia. We have proposed that they can be standardized further by comparing their decline rates at +20 days and +30 days after peak in the rest frame in a manner similar to the Phillips $\Delta m_{15}$ relation. It appears that the fainter the $\mathrm{SN}$, the faster the decline rate. The raw scatter can be reduced to $0.20-0.26$ mag using this $\Delta M_{20}(400)$ relation, depending on which sample and calibration method are used.

We have also uncovered an additional method to standardize the peak magnitudes, by comparing the rest-frame color evolution of SLSN. A comparison of the rate of change of color index $M(400)-M(520)$ with the peak magnitude $M(400)$ shows a tight 
correlation with a very small rms scatter of $0.08-0.13$ mag. The fainter $\mathrm{SNe}$ are redder at peak, and they evolve to redder colors faster than their bluer and brighter counterparts. Although this low rms is encouraging, we caution that it may be due to small numbers of objects because we could only use 8 to 10 of the $\mathrm{SNe}$ due to data limitations. An immediate objective is to increase the sample with enough observational data that this can be tested further.

A difficulty remains in that SLSN Ic may have distinctly different observational (or physical) subclasses. The bulk of the population is split into two broad classes of fast-evolving objects (2005ap-like) and more slowly evolving objects (2007bi-like). It is not immediately clear if these are distinct or if there is a continuum of properties bridging the gap between them. The residuals of the fits in our standardizable candle methods are somewhat dependent on classification of these SLSN into these subclasses. However, the results are not critically dependent on this phenomenological classification. A further complication is the recent discovery of an SLSN that is spectroscopically similar to the SLSN Ic but shows additional weak $\mathrm{H} \alpha$ emission (and hence has been classified as an SLSN type II). Because it may be difficult to deselect this type of SN at high redshift from a pure sample of SLSN Ic, we included this event in our sample to check whether it compromised the results. Although it does appear brighter and bluer than the bulk of the SLSN Ic sample, its inclusion did not significantly increase the scatter in the $\Delta M_{20}(400)$ relation. It did, however, increase the scatter in the peak magnitudecolor evolution calibration to 0.19 dex.

The low volumetric rates of these SLSN mean that they are not useful as primary distance indicators in the local universe $(z<0.1)$. Indeed, the calibration of their peak magnitudes requires an adoption of a cosmology to determine their distance moduli at low to moderate redshift. Their usefulness may be in redshift regimes beyond those possible for SN Ia. With current instrumentation and facilities, they have the potential to probe the universe at $z=2-4$, in which their rest-frame $M(400)$ and $M(520)$ calibration bands correspond to apparent $\mathrm{AB}$ magnitudes of between 23 and 24 in typical near-infrared $J H K$ passbands. They are likely to be detectable out to redshifts of $z \simeq 10$ with future space-based missions such as JWST, EUCLID, and WFIRST.

We thank the anonymous referee for a careful review that improved the clarity of the paper. We wish to thank Mark Sullivan, Bob Nicholl, Andrea Pastorello, Enrico Cappellaro, Anders Jerkstrand, Morgan Fraser, Ting-Wan Chen, David Young, Stuart Sim, Rubina Kotak, and Matt Nicholl for the helpful discussions. C.I. also thanks Robert Quimby, Ragnhild Lunnan, and Stefano Benetti for providing the spectra of SN2005ap, PTF09cnd, PS1-10bzj, and CSS121015. C.I. thanks Fulvio Melia and his team for the careful reading of the paper and their suggestions. The research leading to these results has received funding from the European Research Council under the European Union's Seventh Framework Programme (FP7/20072013)/ERC Grant agreement No. [291222] (PI: S. J. Smartt).

\section{APPENDIX A}

\section{DETAILS OF THE POLYNOMIAL FITTING AND INTERPOLATION OF LIGHT CURVES}

When fitting polynomials to the photometric light curves, we tried as far as possible to use the same time baseline: from $-10 \pm 5$ days before maximum (in the $400 \mathrm{~nm}$ band) to $40 \pm 5$ days after. The fits displayed in Figures 2 and 3 make use of this baseline, and the magnitudes reported in Table 1 are those from interpolation at the specific epochs of peak, +10 days,+20 days, and +30 days, unless otherwise noted (below, we refer to these as the key epochs). Further details on each object are as follows.

1. SN20logx. This is a very well sampled SN light curve with only 2 small gaps around 20 days and 27 days past maximum. We fitted 31 epochs from -8 days prepeak to 40 days after with a third-order polynomial ( $\mathrm{rms}=0.06)$. For the key epochs we used magnitudes from interpolation that were coincident with the measurements available, specifically at epochs 0 and +10 days. A polynomial of the order of three was used to fit the $M(400)-M(520)$ color curve with an rms $=0.05$ in the period -5 days before peak to 40 days after.

2. SN2011kf. We lack specific data close to 2 out of 4 key epochs (+10 days and +30 days), but the light curve is extremely similar to that of SN 2010gx. The light curve is quite well sampled from +40 days onward (Inserra et al. 2013), and we also used these points to constrain the fit. We fitted the 6 epochs available until 45 days postmaximum with a second-order polynomial. There is no noticeable difference between a second- and third-order function. We found an $\mathrm{rms}=0.05$ for the second order. If we would extend the data used out to 60 days, then the difference in the rms fit is less than $2 \sigma$. The magnitudes reported in Table 1 are derived from the fit.

3. SN2011ke. This object has a well-sampled $g$-band light curve in the first 30 days postmaximum. Small gaps exist at +5 days, +17 days, and +27 days after peak. We have measurements specifically at all four key epochs, and the magnitudes from interpolation are in agreement within the fit rms; hence in Table 1 we reported the actual magnitudes. An rms $=0.07$ was found for a third-order polynomial fit to the 19 epochs available for the baseline -17 to 31 days. If we extend the baseline up to 60 days postmaximum and then include the other three epochs, the rms for the same fit order as before increases to 0.08. We also fitted the $M(400)-M(520)$ color evolution with a third-order polynomial ( $\mathrm{rms}=0.07$ ) from -17 days before peak to 31 days after. The rms of the fit does not change if we include the following point at +51 days.

4. SN2012il. For this object, an observed $g$-band magnitude at peak is not specifically available, but we do have an $r$-band measurement exactly at peak. To retrieve a $g$-band peak magnitude, we used our library of template spectra at peak epoch and the observed $r$ magnitude at peak to obtain the $K$ correction to the $400 \mathrm{~nm}$ rest-frame absolute magnitude. To check the reliability of this method, we also fitted the available photometry (from $r$ to $z$ ) around peak epoch with a blackbody and then estimated the $K$ correction factor from $r$ to $400 \mathrm{~nm}$. The values retrieved with these two methods (template and multicolor photometry) were in agreement (see Inserra et al. 2013, for further details on these methods and their application) within an error of $\sim 0.11 \mathrm{mag}$. The peak magnitude in Figure 2 is that from the spectral $K$-corrected method. For the $g$-band data available ( +4 days to +34 days) we used a third-order polynomial fit, obtaining an $\mathrm{rms}=0.04$. The fit displayed in Figure 2 does not use the peak value, but there are measurements close to the key epochs of $+10,+20$, and +30 days. We used the same time baseline of the light curve for the $M(400)-M(520)$ color, 
but we fitted it with a second-order polynomial to avoid overfitting.

5. SN2005ap. We fitted 15 epochs from -5 days from maximum to 26 days after with a third-order polynomial, which resulted in an rms $=0.09$. The point at 19 days past maximum was $4 \sigma$ from this fit, and we removed it during the fitting process in accordance with Chauvenet's criterion.

6. PS1-10ky. This SN did not have a magnitude specifically at peak in the observed $i$ band (chosen for the $400 \mathrm{~nm}$ restframe cross-filter $K$ correction); however, it did have $r$-band measurements. We fitted 15 epochs of $M(400)$ (computed from the observed $i$ band) between +6 and +36 days after maximum with a third-order polynomial $(\mathrm{rms}=0.07)$. The values listed in Table 1 refer to magnitudes from interpolation with this fit. To check the consistency of our fit, we also specifically evaluated the peak magnitude with a $K$ correction from the observed $r$ band at peak to the $400 \mathrm{~nm}$ rest-frame band, together with a spectrophotometric measurement. As in the case of SN2012il, these two methods gave back similar results. The magnitude retrieved is plotted in Figure 2, and it matches the fit well. Hence we are confident that the absolute peak magnitude is reliable. While fitting the $M(400)-M(520)$ color evolution, two points at $\sim 8$ and $\sim 22$ days past maximum were rejected by the application of Chauvenet's criterion (being more than $3 \sigma$ from the fit). The other points in the color evolution were fitted with a fourth-order polynomial with rms $=0.08$.

7. PTF09cnd. The key epochs are available in the observed PTF09cnd light curve. We fitted the epochs available for the -10 days to +40 days baseline with a third-order polynomial with rms $=0.04$. The magnitudes from interpolation are in agreement with the direct measurements, and in Table 1 we report the actual magnitudes.

8. SCP-O6F6. The observed $z$ band was chosen as the filter to $K$ correct to retrieve the $400 \mathrm{~nm}$ band rest frame, and the available data cover out to $\sim 20$ days past maximum. We interpolated the 6 epochs available between -30 days and 20 days from peak with a second- and third-order polynomial, obtaining similar results. The best $\mathrm{rms}=0.05$ was found with the second order. As a consistency check, we also estimated what we would expect to see (assuming our fit is valid) in the $i$-band light curve at $\sim 40$ days because an $i$-band limit at this epoch is reported in Barbary et al. (2009). We find consistency, and our $z$-band fit results would only be incompatible with the limit if the $i$-band mag would be about 1 mag fainter than the limit reported in Barbary et al. (2009). However, no SLSN Ic has published data that show such a steep decrease or dramatic color change at this phase so far.

9. PTF11rks. The faintest object of the sample shows 2 small gaps of 4 and 5 days around 11 days and 23 days past maximum light. We fitted the 14 observations between -3 days and +29 days from peak with a third-order polynomial, obtaining $\mathrm{rms}=0.05$. A polynomial of equivalent order fitted the color evolution on the same baseline with $\mathrm{rms}=$ 0.06 . We noticed that magnitudes from interpolation of the fits extending up to +48 days are in agreement with those retrieved at +48 days by SED fitting. The actual magnitude at peak was in agreement with that from interpolation.

10. PS1-10bzj. The data cover two out of four key epochs, but thanks to sufficient coverage (eight epochs) spanning the baseline range defined above, we retrieved the best fit with a polynomial of third order and $\mathrm{rms}=0.07$. We also fitted the color evolution on the same baseline with a third-order polynomial, retrieving $\mathrm{rms}=0.06$.

11. PS1-10pm. The data (nine epochs) cover from -4 days to +41 days. We fitted them with a third-order polynomial, retrieving $\mathrm{rms}=0.08$.

12. LSQ12dlf. The key epochs are available in the observed LSQ12dlf light curve. We fitted the 30 available epochs for our above-mentioned baseline ( -10 days to 40 days) with a third-order polynomial with $0.09 \mathrm{rms}$. The magnitudes from interpolation are in agreement with the direct measurements, and in Table 1 we report the actual magnitudes. The 12 epochs of the $M(400)-M(520)$ color evolution were fitted with a third-order polynomial and 0.03 as rms.

13. $S N 2013 d g$. We fitted 18 epochs from maximum to 42 days afterward with a third-order polynomial $(\mathrm{rms}=0.06)$. The same number of epochs were fitted for the color evolution, retrieving an $\mathrm{rms}=0.03$.

14. PTF12dam. The light curve is not densely sampled at peak, but we are confident about the fit results and the magnitudes at key epochs because of the slowly evolving light curve. We fitted nine epochs from -15 days to +55 days from peak with a third-order polynomial and an $\mathrm{rms}=0.06$. The color evolution was also fitted in the same period with a third-order polynomial and an rms $=0.04$.

15. PS1-11ap. The data coverage is good with two small gaps around 13 and 25 days after peak. A third-order polynomial was used to fit the 24 data points for $M(400)$ and 20 epochs of color evolution $M(400)-M(520)$ in the range from -16 days to +42 days, retrieving $\mathrm{rms}=0.10$ and rms $=0.11$, respectively. Despite the abundance of data at peak and +30 days from that, we used the magnitudes from interpolation to be consistent through the sample.

16. CSS 121015. We fitted the 24 epochs in the range -15 days to +40 days with a third-order polynomial and $\mathrm{rms}=0.09$. Because the light curve is likely powered by interaction (Benetti et al. 2014), especially around peak, we decided to use the actual peak magnitude instead of that from the fit, and the other values(in Table 1) come from the interpolation. We fit the color evolution in the same range of the light curve, finding an $\mathrm{rms}=0.10$.

\section{APPENDIX B}

\section{TREATMENT OF ERRORS}

When evaluating the errors on our absolute magnitudes, we tried as far as possible to account for all sources of error. As reported in Section 3.2, our observed magnitude in the passband $f$ and absolute magnitude in the passband $400 \mathrm{~nm}$ (the following discussion is valid also for the $520 \mathrm{~nm}$ band) are related as follows:

$$
m_{f}=M(400)+\mu+K_{f \rightarrow 400}+A_{f} .
$$

According to the formalism reported in Blanton \& Roweis (2007), the $K$ correction from $f$ to 400 is the following:

$$
\begin{aligned}
& K_{f \rightarrow 400}=-2.5 \log \left[\frac{1}{1+z}\right. \\
& \left.\quad \times \frac{\int d \lambda_{\mathrm{o}} \lambda_{\mathrm{o}} L_{\lambda}\left(\lambda_{\mathrm{o}} / 1+z\right) f\left(\lambda_{\mathrm{o}}\right) \int d \lambda_{\mathrm{e}} \lambda_{\mathrm{e}} g_{\lambda}^{400}\left(\lambda_{\mathrm{e}}\right) 400\left(\lambda_{\mathrm{e}}\right)}{\int d \lambda_{\mathrm{o}} \lambda_{\mathrm{o}} g_{\lambda}^{f}\left(\lambda_{\mathrm{o}}\right) f\left(\lambda_{\mathrm{o}}\right) \int d \lambda_{\mathrm{e}} \lambda_{\mathrm{e}} L_{\lambda}\left(\lambda_{\mathrm{e}}\right) 400\left(\lambda_{\mathrm{e}}\right)}\right],
\end{aligned}
$$

where $L_{\lambda}$ is the luminosity per unit wavelength, $\lambda_{\mathrm{o}}$ refers to the observed frame, $\lambda_{\mathrm{e}}$ refers to the rest frame (e stands for emitted), 

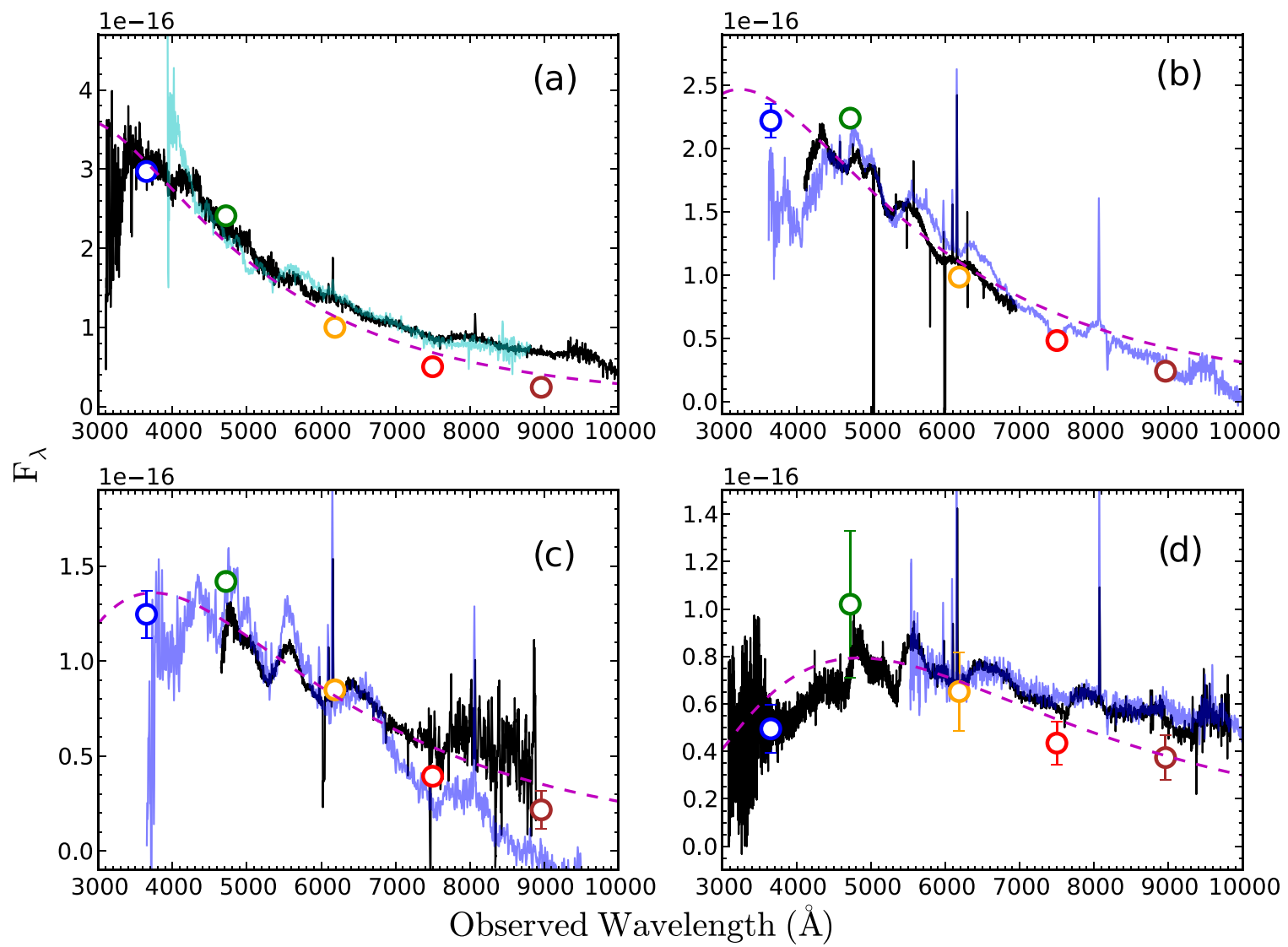

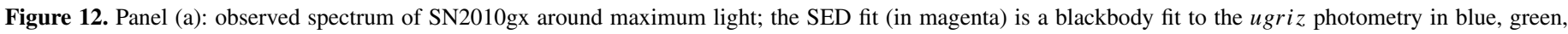

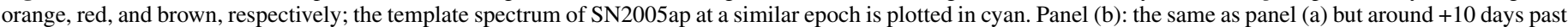

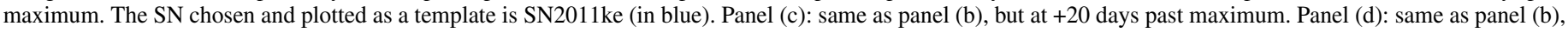
but at +30 days past maximum.

(A color version of this figure is available in the online journal.)

$g_{\lambda}^{400}$ is the flux density per unit wavelength for the $400 \mathrm{~nm}$ band, and $g_{\lambda}^{f}$ is the same for the observed band, and $400(\lambda)$ and $f(\lambda)$ are the response of the instrument per unit photon for the rest and observed filters, respectively. Rewriting Equation (B1), we have

$$
M(400)=m_{f}-\left(5 \log D_{\mathrm{L}}+25\right)-A_{f}-K_{f \rightarrow 400},
$$

where the $K$-correction term is not expanded and the distance modulus has been expressed as a function of distance luminosity $D_{\mathrm{L}}$ in megaparsecs. The errors on $M(400)$, nominally $\sigma_{M(400)}$ and equivalent to the standard deviation, are given by the combination of errors of the terms on the right-hand side of Equation (B3). Assuming as a first approximation that the terms are uncorrelated and the errors deriving from the cosmology adopted are negligible, the errors on our absolute magnitude follow the equation below:

$$
\begin{aligned}
& \sigma_{M(400)}=
\end{aligned}
$$

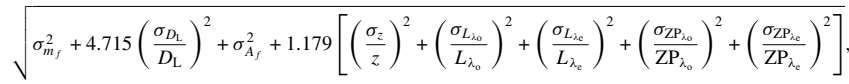

where the differences between the Vega and $\mathrm{AB}$ systems are taken into account by the covariance of the zero points $(\mathrm{ZP})$ of the two systems $\left(\mathrm{ZP}_{\lambda_{0}}\right.$ and $\left.\mathrm{ZP}_{\lambda_{\mathrm{e}}}\right)$. The two major sources of errors are related to observed magnitudes and extinction.
When a cross-filter $K$ correction is made, the errors are usually in the range 0.005-0.05 mag (Kim et al. 1996; Blanton \& Roweis 2007; Hsiao et al. 2007). These values are lower than, or comparable to, those of the errors on extinction and observed magnitudes. During our analysis, we effectively always applied a cross-filter $K$ correction. The errors related to the $K$ correction can be 34\% (0.03-0.04 mag) if we use two different systems for the observed and rest filter band, as we had to do for SN2005ap, PTF09cnd, LSQ12dlf, and CSS121015, and can be up to $0.05 \mathrm{mag}$ if the cross-filter $K$ correction is not applied (Hsiao et al. 2007).

The error treatment so far is applicable if we use the spectrum of the SN to evaluate the $K$ correction. If instead of the actual SN spectrum we use a library template spectrum (of a similar $\mathrm{SN}$ ) or the SED fitting (using blackbody spectra) to observed photometry, then some further scatter is found, but $\sigma_{M(400)}$ is not significantly affected by this. In order to quantitatively investigate the differences between the three methods, we compared the SED photometry fitting method (employing blackbody fits) and the spectral library method (using our 64 spectra) as applied to SN2010gx. We compared these values to those reported in Table 2, which were evaluated using the actual SNe spectra. In Figure 12 the three spectra used for the three methods are illustrated together. In comparison to the $K$-correction values derived with the SNe spectra (reported in Table 2 for SN2010gx), the SED fitting method gives results that are different by $0.01-0.06$ mag depending on the phase and the EW of the emission lines. The library template method results in differences that are 

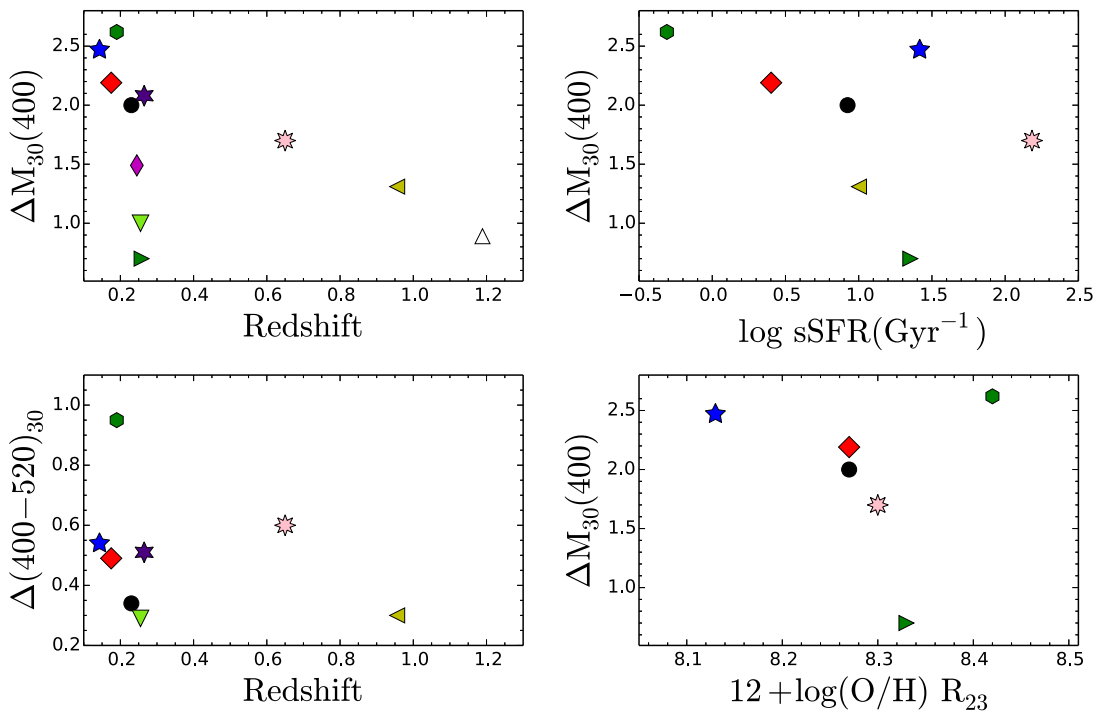

\begin{tabular}{|llll|}
$\bullet$ & SN2010gx & & PTF11rks \\
$\diamond$ & SN2011kf & & SN2011ke \\
$\diamond$ & SN2012il & $\sum_{w}$ & PS1-10bzj \\
$\triangleleft$ & PS1-10ky & $\nabla$ & LSQ12dlf \\
$\triangleright$ & PTF09cnd & $\star$ & SN2013dg \\
$\triangle$ & SCP-06F6 & & \\
\hline
\end{tabular}

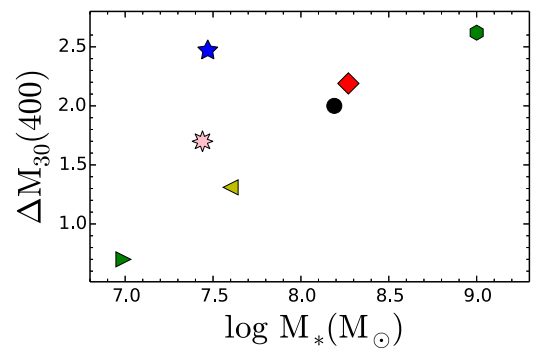

Figure 13. Various plots of example empirical properties of SLSN derived in this paper compared to redshift and host galaxy properties from Chen et al. (2013) and Lunnan et al. (2014).

(A color version of this figure is available in the online journal.)

between 0.01 and $0.05 \mathrm{mag}$, depending on the filter and spectra used as templates. We note that the major differences are found at the extremities of the optical wavelength region (e.g., $u$ and $z$ ). These potential systematic differences were not included in the final estimates of $\sigma_{M(400)}$ (because they are not strictly speaking random errors) but are given here to quantify the differences found when using different methods for $K$ correction.

\section{APPENDIX C}

\section{THE DEPENDENCE OF SLSN PROPERTIES ON THEIR HOST GALAXIES}

In Figure 13 we illustrate an initial comparison of SLSN properties as a function of redshift and host galaxy properties. Two of the empirical properties determined in this paper $\left(\Delta M_{30}(400)\right.$ and $\left.\Delta(400-520)_{30}\right)$ are plotted against redshifts and host galaxy properties from Chen et al. (2013) and Lunnan et al. (2014). The decline parameter after 30 days and the color evolution parameter are representative of the empirical properties we have investigated. The three galaxy properties used are specific star-formation rate, total stellar mass, and oxygen abundance. For oxygen abundance, we took the $R_{23}$ estimate from Lunnan et al. (2014), using the low-metallicity branch value (because the low masses of these SLSN hosts make this branch choice most plausible). As one might expect from the small sample size, there is no clear dependence of SLSN properties on host galaxy properties. The two plots comparing the decline parameters with redshift illustrate that the intrinsically fainter objects are not represented at the higher redshifts, which is not unexpected given the magnitude-limited surveys that the discoveries originate from. One might postulate a trend in the $\Delta M_{30}(400)$ versus $\log M_{\star}$ plot, in the sense that the intrinsically fainter SLSN tend to occur in more massive galaxies. However, this is possibly just driven by one point (either PTF09cnd at the low mass end or PTF11rks at the other mass extreme), and drawing conclusions would not be secure. Thus, an increase in the sample is mandatory in order to better investigate any underlying physical relation or redshift evolution.

\section{REFERENCES}

Agnoletto, I., Benetti, S., Cappellaro, E., et al. 2009, ApJ, 691, 1348 Baltay, C., Rabinowitz, D., Hadjiyska, E., et al. 2013, PASP, 125, 683 Barbary, K., Dawson, K. S., Tokita, K., et al. 2009, ApJ, 690, 1358 Berger, E., Chornock, R., Lunnan, R., et al. 2012, ApJL, 755, L29 Benetti, S., Nicholl, M., Cappellaro, E., et al. 2014, MNRAS, 441, 289 Blanton, M. R., \& Roweis, S. 2007, AJ, 133, 734

Campana, S., Mangano, V., Blustin, A. J., et al. 2006, Natur, 442, 1008 Cano, Z. 2014, arXiv: 1407.2589

Cano, Z., \& Jakobsson, P. 2014, arXiv: 1409.3570

Cao, Y., Kasliwal, M. M., Arcavi, I., et al. 2013, ApJL, 775, L7

Chen, T.-W., Smartt, S. J., Bresolin, F., et al. 2013, ApJL, 763, L28

Chevalier, R. A., \& Irwin, C. M. 2011, ApJL, 729, L6

Chomiuk, L., Chornock, R., Soderberg, A. M., et al. 2011, ApJ, 743, 114

Chornock, R., Berger, E., Rest, A., et al. 2013, ApJ, 767, 162

Cobb, B. E., Bailyn, C. D., van Dokkum, P. G., \& Natarajan, P. 2006, ApJL, 645, L113

Cooke, J., Sullivan, M., Gal-Yam, A., et al. 2012, Natur, 491, 228

D'Andrea, C. B., Sako, M., Dilday, B., et al. 2010, ApJ, 708, 661

Dessart, L., Hillier, D. J., Waldman, R., Livne, E., \& Blondin, S. 2012, MNRAS, 426, L7

Drake, A. J., Djorgovski, S. G., Mahabal, A., et al. 2009, ApJ, 696, 870

Drake, A. J., Djorgovski, S. G., Mahabal, A. A., et al. 2011, ATel, 3343, 1 
Dunkley, J., Komatsu, E., Nolta, M. R., et al. 2009, ApJS, 180, 306 Eisenstein, D. J., Zehavi, I., Hogg, D. W., et al. 2005, ApJ, 633, 560 Foley, R. J., Papenkova, M. S., Swift, B. J., et al. 2003, PASP, 115, 1220

Galama, T. J., Vreeswijk, P. M., van Paradijs, J., et al. 1998, Natur, 395, 670 Gal-Yam, A. 2012, Sci, 337, 927

Gal-Yam, A., Mazzali, P., Ofek, E. O., et al. 2009, Natur, 462, 624

Gehrels, N. 1986, ApJ, 303, 336

Ginzburg, S., \& Balberg, S. 2012, ApJ, 757, 178

Goldhaber, G., Groom, D. E., Kim, A., et al. 2001, ApJ, 558, 359

Guy, J., Astier, P., Baumont, S., et al. 2007, A\&A, 466, 11

Guy, J., Astier, P., Nobili, S., Regnault, N., \& Pain, R. 2005, A\&A, 443, 781

Hamuy, M., Phillips, M. M., Suntzeff, N. B., et al. 1996, AJ, 112, 2438

Hamuy, M., Phillips, M. M., Wells, L. A., \& Maza, J. 1993, PASP, 105, 787

Hamuy, M., \& Pinto, P. A. 2002, ApJL, 566, L63

Hogg, D. W., Baldry, I. K., Blanton, M. R., \& Eisenstein, D. J. 2002, arXiv:astro-ph/0210394

Howell, D. A., Kasen, D., Lidman, C., et al. 2013, ApJ, 779, 98

Hsiao, E. Y., Conley, A., Howell, D. A., et al. 2007, ApJ, 663, 1187

Hunter, D. J., Valenti, S., Kotak, R., et al. 2009, A\&A, 508, 371

Inserra, C., Smartt, S. J., Fraser, M., et al. 2012, ATel, 4329, 1

Inserra, C., Smartt, S. J., Jerkstrand, A., et al. 2013, ApJ, 770, 128

Kaiser, N., Burgett, W., Chambers, K., et al. 2010, Proc. SPIE, 7733, 77330E

Kasen, D., \& Bildsten, L. 2010, ApJ, 717, 245

Kim, A., Goobar, A., \& Perlmutter, S. 1996, PASP, 108, 190

Komatsu, E., Smith, K. M., Dunkley, J., et al. 2011, ApJS, 192, 18

Kostrzewa-Rutkowska, Z., Kozłowski, S., Wyrzykowski, Ł., et al. 2013, ApJ, 778,168

King, A. L., Davis, T. M., Denney, K. D., Vestergaard, M., \& Watson, D. 2014, MNRAS, 441, 3454

Laureijs, R., Amiaux, J., Arduini, S., et al. 2011, arXiv:1110.3193

Leloudas, G., Chatzopoulos, E., Dilday, B., et al. 2012, A\&A, 541, A129

Lord, S. D. 1992, NASA Technical Memorandum 103957

Lunnan, R., Chornock, R., Berger, E., et al. 2013, ApJ, 771, 97

Lunnan, R., Chornock, R., Berger, E., et al. 2014, ApJ, 787, 138

Maguire, K., Kotak, R., Smartt, S. J., et al. 2010, MNRAS, 403, L11

Mandel, K. S., Narayan, G., \& Kirshner, R. P. 2011, ApJ, 731, 120

Mandel, K. S., Wood-Vasey, W. M., Friedman, A. S., \& Kirshner, R. P. 2009, ApJ, 704, 629

Mazzali, P. A., Valenti, S., Della Valle, M., et al. 2008, Sci, 321, 1185

McCrum, M., Smartt, S. J., Kotak, R., et al. 2014a, MNRAS, 437, 656

McCrum, M., Smartt, S. J., Rest, A., et al. 2014b, arXiv:1402.1631

McKenzie, E. H., \& Schaefer, B. E. 1999, PASP, 111, 964

Mirabal, N., Halpern, J. P., An, D., Thorstensen, J. R., \& Terndrup, D. M 2006, ApJL, 643, L99

Modjaz, M., Li, W., Butler, N., et al. 2009, ApJ, 702, 226

Nicholl, M., Smartt, S. J., Jerkstrand, A., et al. 2013, Natur, 502, 346

Nicholl, M., Smartt, S. J., Jerkstrand, A., et al. 2014, MNRAS, 444, 2096

Nugent, P., Kim, A., \& Perlmutter, S. 2002, PASP, 114, 803

Nugent, P., Sullivan, M., Ellis, R., et al. 2006, ApJ, 645, 841

Oke, J. B., \& Sandage, A. 1968, ApJ, 154, 2
Pandey, S. B., Anupama, G. C., Sagar, R., et al. 2003, MNRAS, 340, 375

Pastorello, A., Kasliwal, M. M., Crockett, R. M., et al. 2008, MNRAS, 389,955

Pastorello, A., Smartt, S. J., Botticella, M. T., et al. 2010a, ApJL, 724, L16

Pastorello, A., Smartt, S. J., Kankare, D., et al. 2010b, ATel, 2504, 1

Patat, F., Cappellaro, E., Danziger, J., et al. 2001, ApJ, 555, 900

Patat, F., Chandra, P., Chevalier, R., et al. 2007, Sci, 317, 924

Percival, W. J., Reid, B. A., Eisenstein, D. J., et al. 2010, MNRAS, 401, 2148

Perlmutter, S., Gabi, S., Goldhaber, G., et al. 1997, ApJ, 483, 565

Phillips, M. M. 1993, ApJL, 413, L105

Pian, E., Mazzali, P. A., Masetti, N., et al. 2006, Natur, 442, 1011

Poznanski, D., Butler, N., Filippenko, A. V., et al. 2009, ApJ, 694, 1067

Prieto, J. L., Drake, A. J., Mahabal, A. A., et al. 2012, ATel, 3883, 1

Prieto, J. L., Rest, A., \& Suntzeff, N. B. 2006, ApJ, 647, 501

Pskovskii, I. P. 1977, SvA, 21, 675

Quimby, R. 2006, CBET, 644, 1

Quimby, R. M., Aldering, G., Wheeler, J. C., et al. 2007, ApJL, 668, L99

Quimby, R. M., Castro, F., Gerardy, C. L., et al. 2005, BAAS, 37, 171.02

Quimby, R. M., Gal-Yam, A., Arcavi, I., et al. 2011a, ATel, 3841, 1

Quimby, R. M., Kulkarni, S. R., Kasliwal, M. M., et al. 2011b, Natur, 474, 487

Quimby, R. M., Kulkarni, S. R., Ofek, E., et al. 2010, ATel, 2492, 1

Quimby, R. M., Oguri, M., More, A., et al. 2014, Sci, 344, 396

Quimby, R. M., Sternberg, A., \& Matheson, T. 2011c, ATel, 3344, 1

Quimby, R. M., Werner, M. C., Oguri, M., et al. 2013a, ApJL, 768, L20

Quimby, R. M., Yuan, F., Akerlof, C., \& Wheeler, J. C. 2013b, MNRAS, 431, 912

Rau, A., Kulkarni, S. R., Law, N. M., et al. 2009, PASP, 121, 1334

Richmond, M. W., van Dyk, S. D., Ho, W., et al. 1996, AJ, 111, 327

Riess, A. G., Filippenko, A. V., Challis, P., et al. 1998, AJ, 116, 1009

Riess, A. G., Press, W. H., \& Kirshner, R. P. 1996, ApJ, 473, 88

Rodney, S. A., Riess, A. G., Dahlen, T., et al. 2012, ApJ, 746, 5

Rust, B. W. 1974, BAAS, 6, 309

Smith, N., Li, W., Foley, R. J., et al. 2007, ApJ, 666, 1116

Smith, N., \& McCray, R. 2007, ApJL, 671, L17

Soderberg, A. M., Berger, E., Page, K. L., et al. 2008, Natur, 453, 469

Sollerman, J., Jaunsen, A. O., Fynbo, J. P. U., et al. 2006, A\&A, 454, 503

Sollerman, J., Kozma, C., Fransson, C., et al. 2000, ApJL, 537, L127

Stritzinger, M., Mazzali, P., Phillips, M. M., et al. 2009, ApJ, 696, 713

Suzuki, N., Rubin, D., Lidman, C., et al. 2012, ApJ, 746, 85

Tanaka, M., Moriya, T. J., Yoshida, N., \& Nomoto, K. 2012, MNRAS, 422, 2675

Tomita, H., Deng, J., Maeda, K., et al. 2006, ApJ, 644, 400

Tonry, J. L., Stubbs, C. W., Lykke, K. R., et al. 2012, ApJ, 750, 99

Valenti, S., Benetti, S., Cappellaro, E., et al. 2008a, MNRAS, 383, 1485

Valenti, S., Elias-Rosa, N., Taubenberger, S., et al. 2008b, ApJL, 673, L155

Valenti, S., Fraser, M., Benetti, S., et al. 2011, MNRAS, 416, 3138

Woosley, S. E. 2010, ApJL, 719, L204

Yoshii, Y., Tomita, H., Kobayashi, Y., et al. 2003, ApJ, 592, 467

Young, D. R., Smartt, S. J., Valenti, S., et al. 2010, A\&A, 512, A70 


\title{
ERRATUM: "SUPERLUMINOUS SUPERNOVAE AS STANDARDIZABLE CANDLES AND HIGH-REDSHIFT DISTANCE PROBES” (2014, ApJ, 796, 87)
}

\author{
C. INSERRA AND S. J. SMARTT \\ Astrophysics Research Centre, School of Mathematics and Physics, Queens University Belfast, Belfast BT7 1NN, UK; c.inserra@qub.ac.uk \\ Received 2015 May 15; accepted 2015 May 29; published 2015 July 6
}

The authors regret that in the published version of this article there were calculation errors in many of the values in Table 1 and in particular the important values for $M(400)$ and the decline rates. These errors were mostly, but not exclusively, due to a sign error in the application of the $K$-corrections. These errors unfortunately propagate through many of the subsequent tables and figures with Figures 2-11 and 13 being affected to various degrees (some important, some trivial). The corrected values in Table 1 are reported here along with the updated versions of the appropriate tables and figures (Figures 1-6). The two main conclusions of the paper (that a peak magnitude-decline rate relation and a peak magnitude-color evolution relation exist) are unchanged, but the quantitative rms values are larger than previously reported. The new values of the linear fits along with the rms and Spearman/Pearson coefficients are now given in an updated version of Table 3 in this erratum. We have also corrected a few typographical errors that were present in Table 2 of the published version.

These corrections to the values in Table 1 mean that the peak absolute magnitudes of the pure sample result in a mean absolute magnitude and standard deviation of of $M(400)=-21.64 \pm 0.46$. If the two slowly declining, 2007bi-like SLSN are included, then the extended sample mean and standard deviation are $M(400)=-21.65 \pm 0.43$. The complete sample then has corrected values of $M(400)=-21.70 \pm 0.46$.

A further consequence of the new values now calculated in Table 1 are revised numbers in Section 4 where we calculated the peak magnitude-decline relation over the 30 day baseline, $\Delta M_{30}(400)$, excluding PTF11rks and PS1-10bzj from the pure sample. The corrections mean that this exclusion results in an rms of \pm 0.14 , a Spearman rank-order correlation coefficient of 0.95 and the Pearson's $r$ test gives $r=0.91$. Thus the conclusions are unchanged in that the exclusion improves the rms and correlation statistics.

Table 1

Sample of SLSN

\begin{tabular}{|c|c|c|c|c|c|c|c|c|c|c|c|}
\hline SN & $z$ & $\mathrm{E}(\mathrm{B}-\mathrm{V})$ & $m$ & Ref. $^{\text {a }}$ & Filters 400,520 & $A_{f}$ & $M(400)$ & $\Delta M_{10}(400)$ & $\Delta M_{20}(400)$ & $\Delta M_{30}(400)$ & $\Delta \mathrm{M}(400-520)_{30}$ \\
\hline \multicolumn{12}{|c|}{ SLSN Ic (2005ap-like) } \\
\hline SN2011ke & 0.143 & 0.01 & $17.70(\mathrm{~g})$ & 1 & $\mathrm{~g} \rightarrow 400, \mathrm{r} \rightarrow 520$ & 0.05 & -21.27 & 0.46 & 1.22 & 2.14 & 0.65 \\
\hline SN2012il & 0.175 & 0.02 & $18.34(\mathrm{~g})^{*}$ & 1 & $\mathrm{~g} \rightarrow 400, \mathrm{r} \rightarrow 520$ & 0.09 & -21.31 & 0.41 & 1.06 & 1.74 & 0.50 \\
\hline PTF11rks & 0.190 & 0.04 & $19.13(\mathrm{~g})$ & 1 & $\mathrm{~g} \rightarrow 400, \mathrm{r} \rightarrow 520$ & 0.17 & -20.63 & 0.28 & 0.95 & 2.02 & 0.95 \\
\hline SN2010gx & 0.230 & 0.04 & $18.43(\mathrm{~g})$ & 2 & $\mathrm{~g} \rightarrow 400, \mathrm{r} \rightarrow 520$ & 0.13 & -21.73 & 0.13 & 0.63 & 1.41 & 0.58 \\
\hline SN2011kf & 0.245 & 0.02 & $18.60(\mathrm{~g})$ & 1 & $\mathrm{~g} \rightarrow 400$ & 0.09 & -21.76 & 0.09 & 0.54 & 1.25 & $\cdots$ \\
\hline LSQ12dlf & 0.255 & 0.01 & $18.78(\mathrm{~V})$ & 3 & $\mathrm{~V} \rightarrow 400, \mathrm{R} \rightarrow 520$ & 0.03 & -21.59 & 0.38 & 0.73 & 1.34 & 0.55 \\
\hline PTF09cnd & 0.258 & 0.02 & $18.09(\mathrm{R})$ & 4 & $\mathrm{R} \rightarrow 400$ & 0.05 & -22.15 & 0.19 & 0.50 & 0.94 & $\cdots$ \\
\hline SN2013dg & 0.265 & 0.04 & $19.26(\mathrm{~g})^{*}$ & 3 & $\mathrm{~g} \rightarrow 400, \mathrm{r} \rightarrow 520$ & 0.15 & -21.38 & 0.43 & 1.24 & 2.00 & 0.75 \\
\hline SN2005ap & 0.283 & 0.01 & $18.35(\mathrm{R})$ & 5 & $\mathrm{R} \rightarrow 400$ & 0.02 & -22.12 & 0.19 & 0.66 & $\cdots$ & $\cdots$ \\
\hline PS1-10bzj & 0.650 & 0.01 & $21.44(\mathrm{r})^{*}$ & 6 & $r \rightarrow 400, z \rightarrow 520$ & 0.02 & -21.11 & 0.21 & 0.81 & 1.55 & 0.66 \\
\hline PS1-10ky & 0.956 & 0.03 & $21.27(\mathrm{i})$ & 7 & $\mathrm{i} \rightarrow 400, \mathrm{z} \rightarrow 520$ & 0.06 & -22.07 & 0.21 & 0.90 & 1.31 & 0.31 \\
\hline SCP-06F6 & 1.189 & 0.01 & $21.04(\mathrm{z})$ & 8 & $\mathrm{z} \rightarrow 400$ & 0.01 & -22.17 & 0.11 & 0.44 & 0.90 & $\ldots$ \\
\hline PS1-10pm & 1.206 & 0.02 & $21.74(\mathrm{z})$ & 9 & $z \rightarrow 400$ & 0.02 & -22.03 & 0.15 & 0.43 & $\cdots$ & $\cdots$ \\
\hline \multicolumn{12}{|c|}{ SLSN Ic (2007bi-like) } \\
\hline PTF12dam & 0.107 & 0.01 & $16.84(\mathrm{~g})^{*}$ & 10 & $\mathrm{~g} \rightarrow 400, \mathrm{r} \rightarrow 520$ & 0.04 & -21.56 & 0.02 & 0.15 & 0.28 & 0.08 \\
\hline PS1-11ap & 0.524 & 0.01 & $20.11(\mathrm{r})$ & 11 & $\mathrm{r} \rightarrow 400, \mathrm{i} \rightarrow 520$ & 0.02 & -21.82 & 0.09 & 0.28 & 0.60 & 0.11 \\
\hline \multicolumn{12}{|c|}{ SLSN II } \\
\hline CSS121015 & 0.287 & 0.08 & $18.19(\mathrm{~V})$ & 12 & $\mathrm{~V} \rightarrow 400, \mathrm{R} \rightarrow 520$ & 0.24 & -22.48 & 0.25 & 0.76 & 0.93 & 0.21 \\
\hline
\end{tabular}

Note. From left to right : SN designation; measured redshift; foreground extinction; observed magnitude (AB system) at peak with the band used in parentheses; reference; observed filters used to calculate the synthetic $400 \mathrm{~nm}$ and $520 \mathrm{~nm}$ magnitudes; extinction toward the SN in the observed band; absolute magnitude in the $400 \mathrm{~nm}$ band, magnitude decrease in 10, 20, and 30 days; the color change between the $400 \mathrm{~nm}$ and $520 \mathrm{~nm}$ synthetic bands at peak and 30 days after.

${ }^{a}$ References: 1. Inserra et al. (2013), 2. Pastorello et al. (2010), 3. Nicholl et al. (2014), 4. Quimby et al. (2011), 5. Quimby et al. (2007), 6. Lunnan et al. (2013), 7. Chomiuk et al. (2011), 8. Barbary et al. 2009, 9. McCrum et al. (2014), 10. Nicholl et al. (2013), 11. McCrum et al. (2015), 12. Benetti et al. (2014).

* The published magnitude closest in time to the peak of the polynomial fit. 
Table 2

Calculated $K$-Corrections for the Sample of SLSN

\begin{tabular}{|c|c|c|c|c|c|c|c|}
\hline SN & $z$ & $K_{f \rightarrow 400}^{\text {peak }}$ & $K_{f \rightarrow 400}^{10}$ & $K_{f \rightarrow 400}^{20}$ & $K_{f \rightarrow 400}^{30}$ & $K_{f \rightarrow 520}^{\text {peak }}$ & $K_{f \rightarrow 520}^{30}$ \\
\hline \multicolumn{8}{|c|}{ SLSN Ic (2005ap-like) } \\
\hline SN2011ke & 0.143 & $-0.18(\mathrm{P})$ & $-0.17(\mathrm{~S})$ & $-0.22(\mathrm{~S})$ & $-0.24(\mathrm{P})$ & $-0.11(\mathrm{~S})$ & $-0.16(\mathrm{~S})$ \\
\hline PTF11rks & 0.190 & $-0.19(\mathrm{~S})$ & $-0.17(\mathrm{~S})$ & $-0.16(\mathrm{~S})$ & $-0.17(\mathrm{P})$ & $-0.19(\mathrm{~S})$ & $-0.19(\mathrm{P})$ \\
\hline SN2010gx & 0.230 & $-0.23(\mathrm{~S})$ & $-0.18(\mathrm{~S})$ & $-0.18(\mathrm{~S})$ & $-0.07(\mathrm{~S})$ & $-0.23(\mathrm{~S})$ & $-0.20(\mathrm{~S})$ \\
\hline SN2011kf & 0.245 & $-0.13(\mathrm{~L})$ & $-0.10(\mathrm{~L})$ & $-0.08(\mathrm{~S})$ & $-0.08(\mathrm{~S})$ & $\ldots$ & $\ldots$ \\
\hline SN2013dg & 0.265 & $-0.30(\mathrm{P})$ & $-0.25(\mathrm{~S})$ & $-0.40(\mathrm{~S})$ & $-0.47(\mathrm{~S})$ & $-0.45(\mathrm{~S})$ & $-0.40(\mathrm{~S})$ \\
\hline SN2005ap & 0.283 & $-0.33(\mathrm{~S})$ & $-0.57(\mathrm{~L})$ & $-0.60(\mathrm{~L})$ & $\ldots$ & $\ldots$ & $\ldots$ \\
\hline PS1-10bzj & 0.650 & $-0.58(\mathrm{P})$ & $-0.57(\mathrm{~S})$ & $-0.46(\mathrm{~S})$ & $-0.48(\mathrm{~L})$ & $-0.37(\mathrm{P})$ & $-0.53(\mathrm{~L})$ \\
\hline PS1-10ky & 0.956 & $-0.73(\mathrm{~S})$ & $-0.75(\mathrm{P})$ & $-0.76(\mathrm{P})$ & $-0.68(\mathrm{~S})$ & $-0.65(\mathrm{P})$ & $-0.59(\mathrm{P})$ \\
\hline SCP-06F6 & 1.189 & $-1.35(\mathrm{~L})$ & $-1.45(\mathrm{~L})$ & $-1.51(\mathrm{~L})$ & $-1.56(\mathrm{~L})$ & $\ldots$ & $\cdots$ \\
\hline PS1-10pm & 1.206 & $-0.84(\mathrm{P})$ & $-0.96(\mathrm{P})$ & $-1.04(\mathrm{P})$ & $\ldots$ & $\cdots$ & $\cdots$ \\
\hline \multicolumn{8}{|c|}{ SLSN II } \\
\hline CSS121015 & 0.287 & $-0.37(\mathrm{~S})$ & $-0.36(\mathrm{~S})$ & $-0.35(\mathrm{~S})$ & $-0.32(\mathrm{P})$ & $-0.40(\mathrm{~S})$ & $-0.22(\mathrm{P})$ \\
\hline
\end{tabular}

Note. $(\mathrm{S})$ denotes that a spectrum of the same object has been used to evaluate the $K$-correction. $(\mathrm{P})$ indicates that the multi-color photometry of the object was used, while (L) means that we used our library of spectra.

Table 3

Fit Parameters and Statistical Results of our Pure, Extended, and Complete Sample

\begin{tabular}{|c|c|c|c|c|c|c|}
\hline Days & $\mathrm{N}$ (objects) & a & $\mathrm{b}$ & $\sigma(\mathrm{mag})$ & Spearman & Pearson \\
\hline \multicolumn{7}{|c|}{$\Delta \mathrm{M}_{\text {day }}(400)$ pure sample } \\
\hline 10 & 13 & $2.06(1.17)$ & $-22.15(0.29)$ & 0.38 & 0.65 & 0.56 \\
\hline 20 & 13 & $1.19(0.47)$ & $-22.53(0.37)$ & 0.34 & 0.73 & 0.66 \\
\hline 30 & 11 & $0.95(0.23)$ & $-22.99(0.35)$ & 0.25 & 0.90 & 0.84 \\
\hline \multicolumn{7}{|c|}{$\Delta M(400-520)$ pure sample } \\
\hline 30 & 8 & $2.02(0.42)$ & $-22.64(0.26)$ & 0.19 & 0.71 & 0.88 \\
\hline \multicolumn{7}{|c|}{$\Delta \mathrm{M}_{\text {day }}(400)$ extended sample } \\
\hline 10 & 15 & $1.57(0.99)$ & $-22.00(0.22)$ & 0.38 & 0.51 & 0.49 \\
\hline 20 & 15 & $0.73(0.37)$ & $-22.16(0.26)$ & 0.36 & 0.58 & 0.54 \\
\hline 30 & 13 & $0.50(0.20)$ & $-22.26(0.27)$ & 0.33 & 0.75 & 0.64 \\
\hline \multicolumn{7}{|c|}{$\Delta M(400-520)$ extended sample } \\
\hline 30 & 10 & $1.09(0.45)$ & $-22.00(0.23)$ & 0.26 & 0.70 & 0.74 \\
\hline \multicolumn{7}{|c|}{$\Delta \mathrm{M}_{\text {day }}(400)$ complete sample } \\
\hline 10 & 16 & $1.49(1.10)$ & $-22.03(0.25)$ & 0.42 & 0.42 & 0.42 \\
\hline 20 & 16 & $0.71(0.42)$ & $-22.20(0.30)$ & 0.41 & 0.51 & 0.47 \\
\hline 30 & 14 & $0.57(0.22)$ & $-22.39(0.28)$ & 0.36 & 0.74 & 0.64 \\
\hline \multicolumn{7}{|c|}{$\Delta M(400-520)$ complete sample } \\
\hline 30 & 11 & $1.34(0.42)$ & $-22.19(0.20)$ & 0.31 & 0.71 & 0.75 \\
\hline
\end{tabular}

Note. Least-squares fits for an unweighted linear fit of the form $M_{\max }(400)=a \Delta M_{\text {day }}(400)+b$ with uncertainties in parentheses. The $\sigma$ is the standard deviation of this fit. The last column gives the Spearman rank-order correlation coefficient and the Pearson correlation coefficient $r$. As the errors on each value of $M(400)$ are fairly similar, a weighted calculation is not significantly different. For completeness, we have reported the fit values obtained excluding CSS121015; although SN2005ap and PS1-10pm are left out of the 30 day decline calculations as they do not have enough data. 

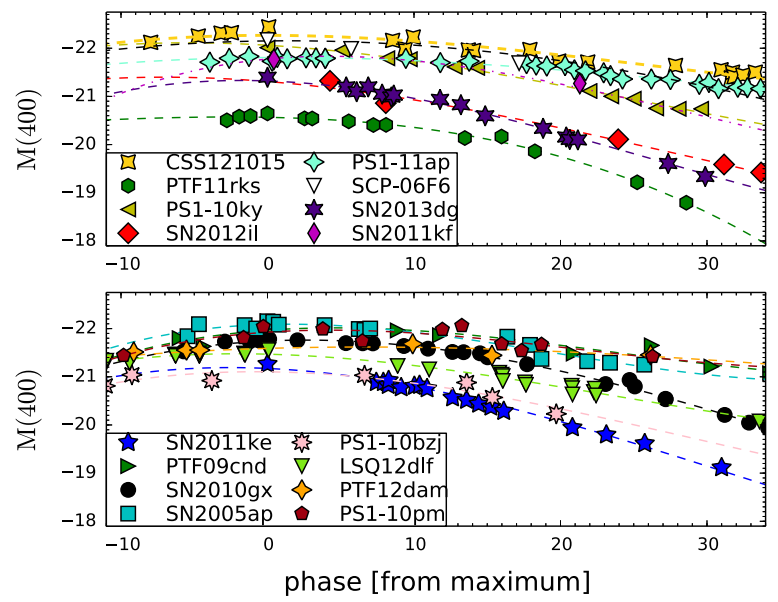

Figure 1. Light curves of our SLSN Ic sample listed in Table 1. The absolute magnitudes, $M(400)$, are for the $400 \mathrm{~nm}$ band after $K$-correction. Time dilation has been applied to report the phase in rest-frame days with respect to maximum light. Polynomial fits to the photometry of the $16 \mathrm{SNe}$ are shown as broken lines. The line fits are all third order polynomials, apart from SN2011kf and SCP06F6 (second order polynomials) and CSS121015 (fourth order polynomial). The objects are split into two panels simply for clarity and illustration purposes.

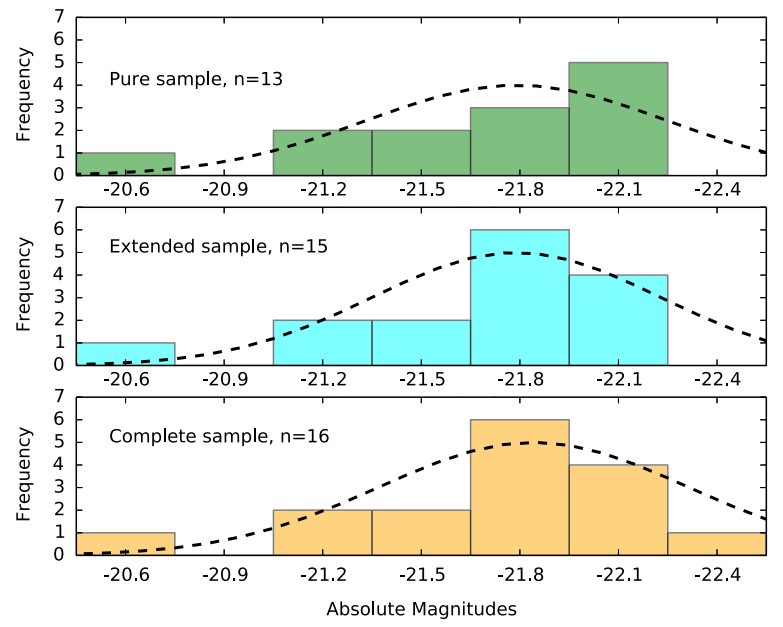

Figure 2. Histograms of the raw (uncorrected) absolute peak magnitudes, $M(400)$, for the three samples defined in the original paper. The dashed lines are least-squares, best-fit Gaussians. Assuming confidence limits for small number events from Gehrels (1986), the Gaussian fits all comfortably fall within the $1 \sigma$ errors of the frequencies.
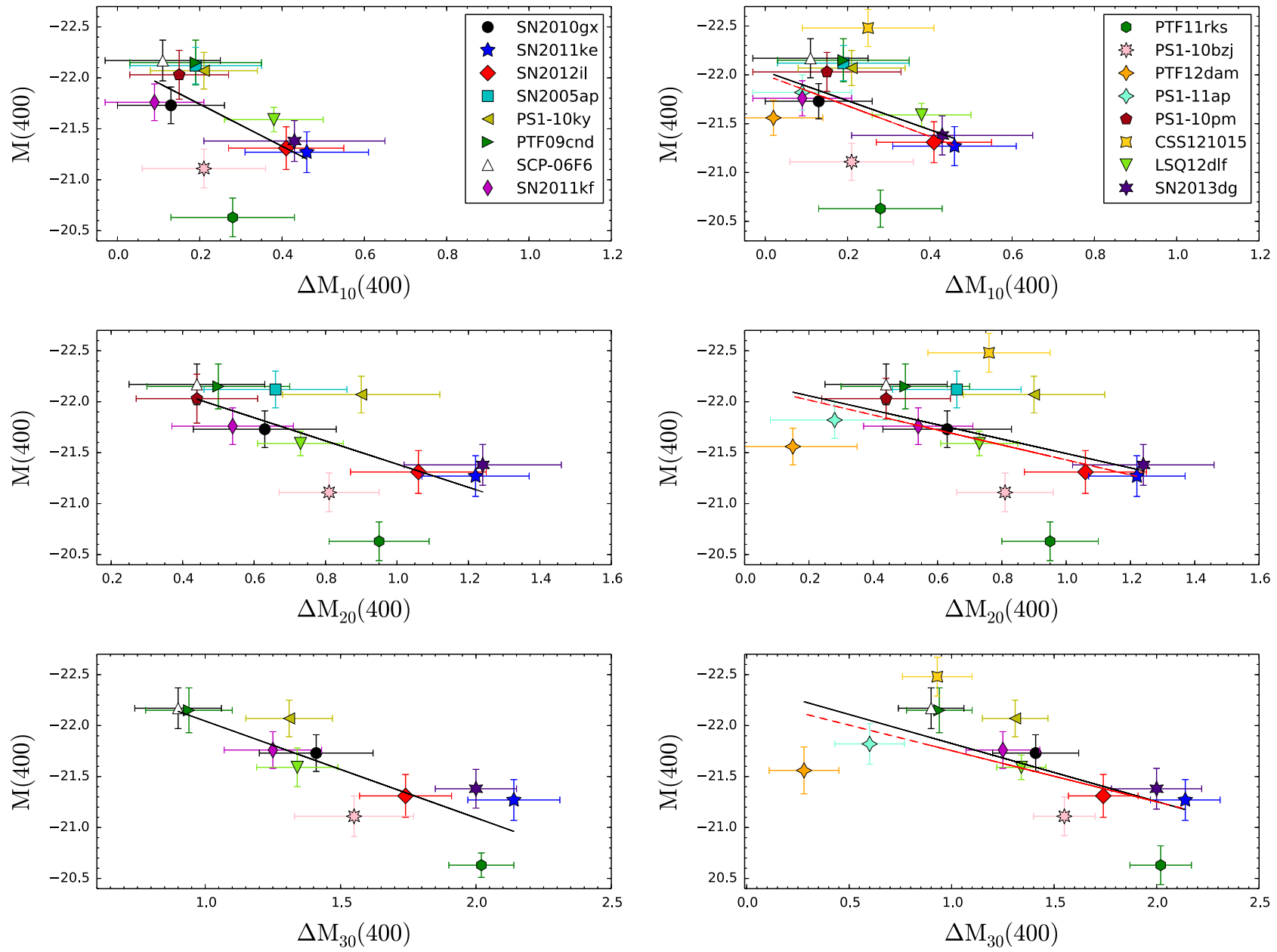

Figure 3. Peak magnitude-decline rate relation. The absolute peak magnitude in the $400 \mathrm{~nm}$ band, $M(400)$, is plotted vs $\Delta \mathrm{M}_{10}(400), \Delta \mathrm{M}_{20}(400)$, and $\Delta \mathrm{M}_{30}(400)$. The latter three values are measures of the light-curve decline (in magnitudes) during the first 10, 20, and 30 days after maximum. The left-hand column of plots shows only the 2005ap-like events (pure sample) and the right-hand column shows all SLSN. The dashed-red line is the fit of the extended sample, while the black of the complete sample.The $\mathrm{SN}$ magnitudes and the corresponding $\Delta \mathrm{M}_{\mathrm{day}}(400)$ are reported in Table 1, while the parameters of the linear regression fits (black line) are listed in Table 3. 

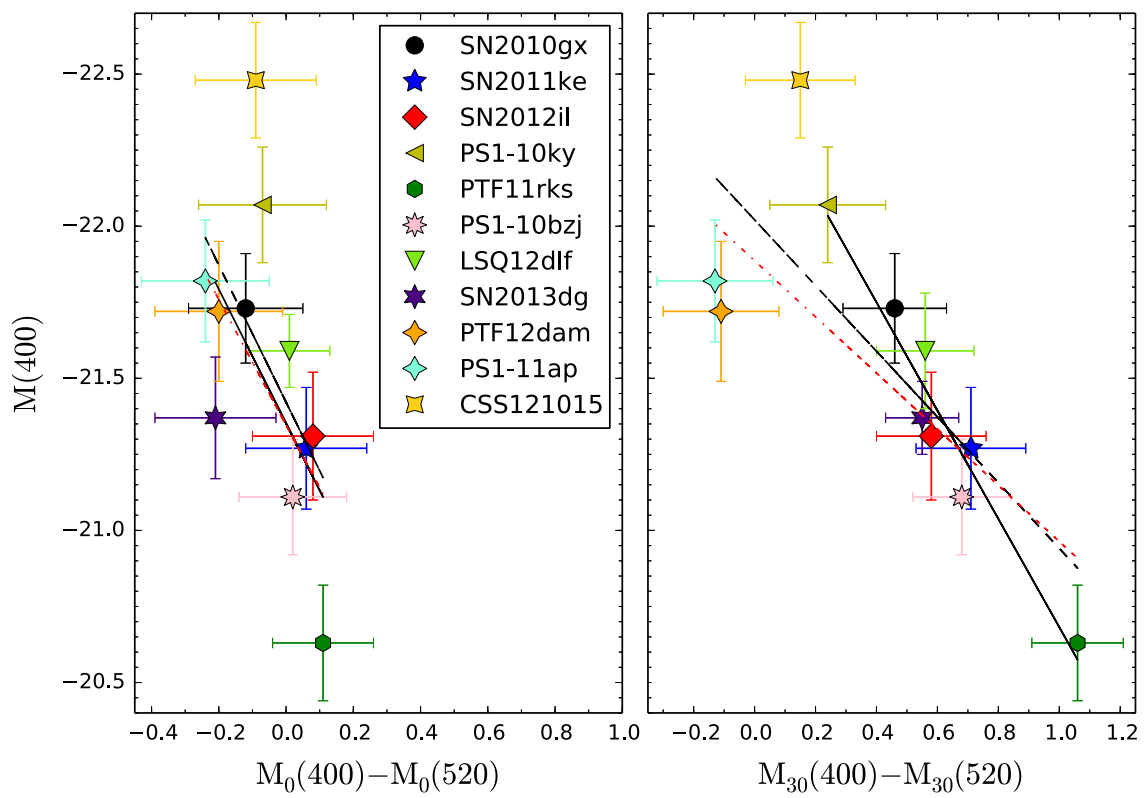

Figure 4. Peak magnitude-color evolution relation. This plot illustrates the apparent dependency of peak magnitude on the color and color evolution rate. The left panel plots the $M(400)$ peak magnitude vs the color at peak brightness. The right-panel plots the same $M(400)$ at peak versus the color at +30 days (rest frame). The black line fit refers only to the pure sample (2005ap-like objects), the red dot-dashed line fits the extended sample (which includes the 2007bi-like objects) while the black dashed line fits the complete sample.
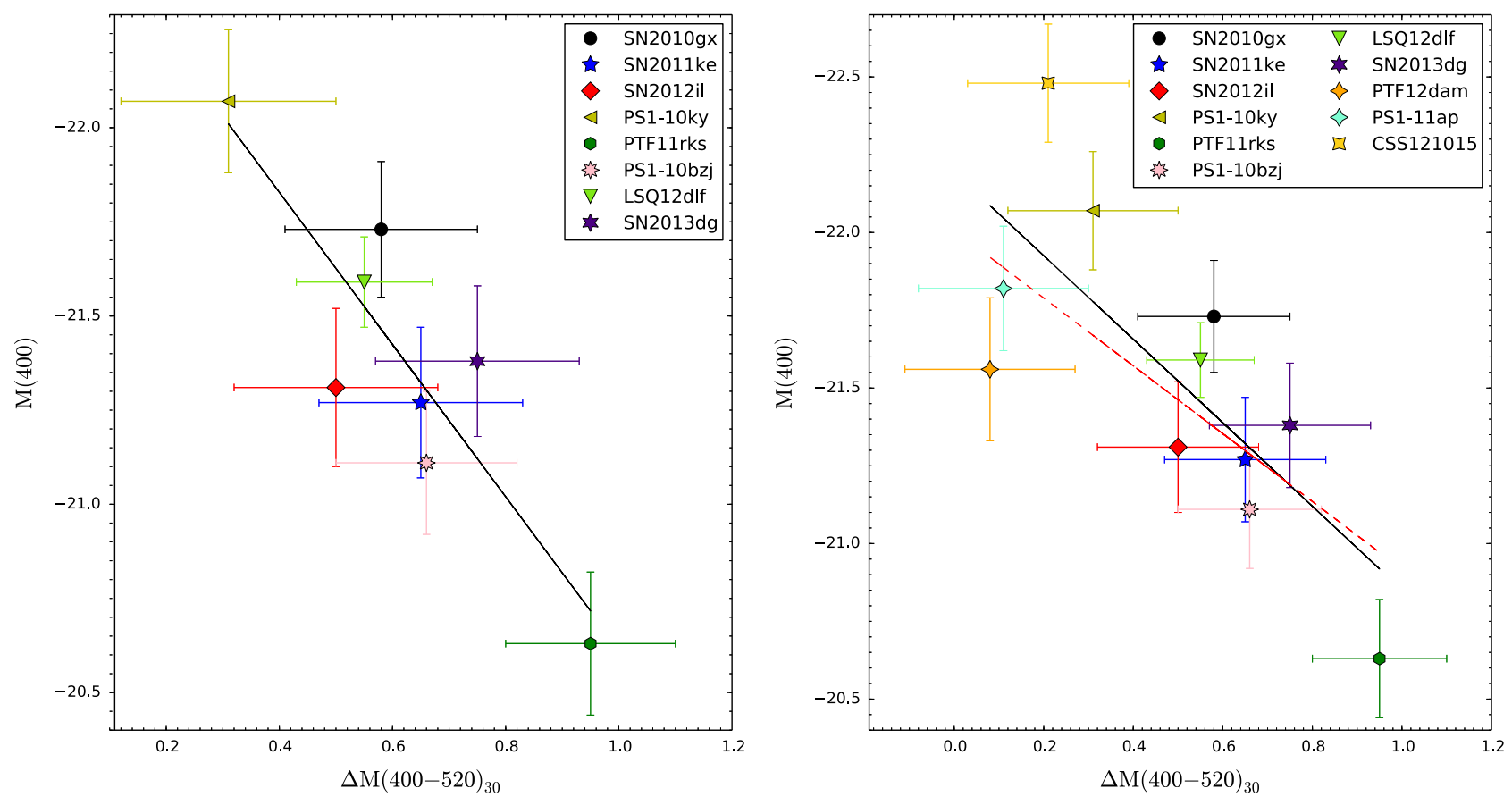

Figure 5. Peak magnitude-color evolution relation. Absolute peak magnitudes in the $400 \mathrm{~nm}$ band, $M(400)$, vs the color change from peak to +30 days (rest frame). We define $\Delta M(400-520)_{30}=\left(M_{0}(400)-M_{0}(520)\right)-\left(M_{30}(400)-M_{30}(520)\right)$ The bands at $400 \mathrm{~nm}$ and $520 \mathrm{~nm}$ are defined in the original paper, and the values for $\Delta M(400-520)_{30}$ values are reported in Table 1. Left: a plot of 8 of the pure sample which have the necessary bands covered in the observer frame to calculate this color term. Right: 11 objects of the complete sample which have the necessary data. The black lines are least-squares straight line fits to all data in each plot. The red dotted line leaves out CSS121015. The parameters of these line fits are are listed in Table 3. 

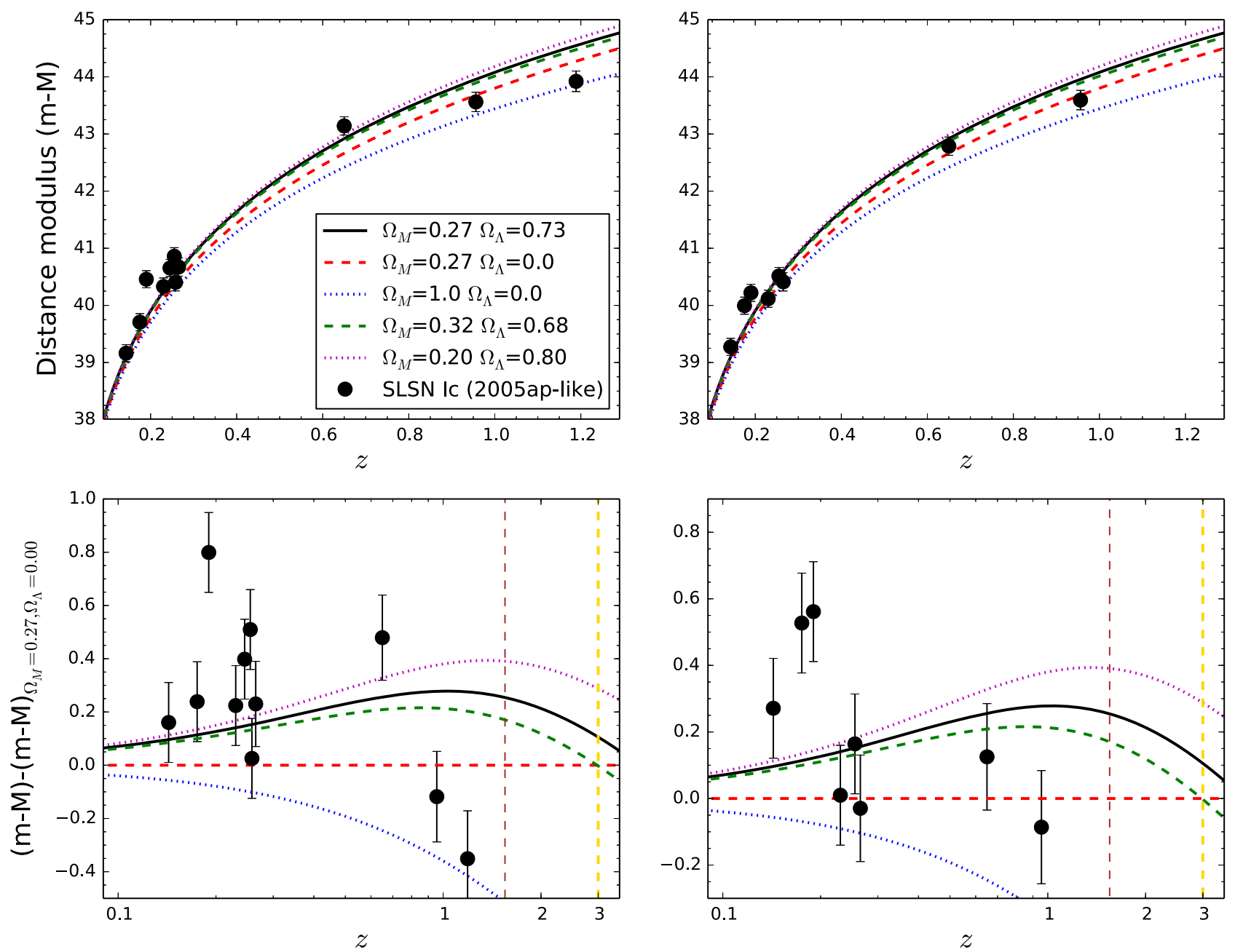

Figure 6. Hubble diagrams for SLSN Ic. Different cosmologies are plotted in comparison with that chosen (solid black line) to measure the distance moduli of the sample presented. Left panels: the distance moduli measured using the $\Delta M_{30}$ decline relation. Right panels: the distance moduli measured using the peak magnitudecolor decline relation. The lower panels show the residuals of the distances relative to a $\Omega_{\mathrm{M}}=0.27, \Omega_{\Lambda}=0$ universe. The brown, dashed vertical line represents the SNe Ia upper limit with the current generation of telescopes (ground and space), whereas the gold, dashed line represents an approximate limit for discovery and monitoring of SLSN Ic with the current generation of telescopes.

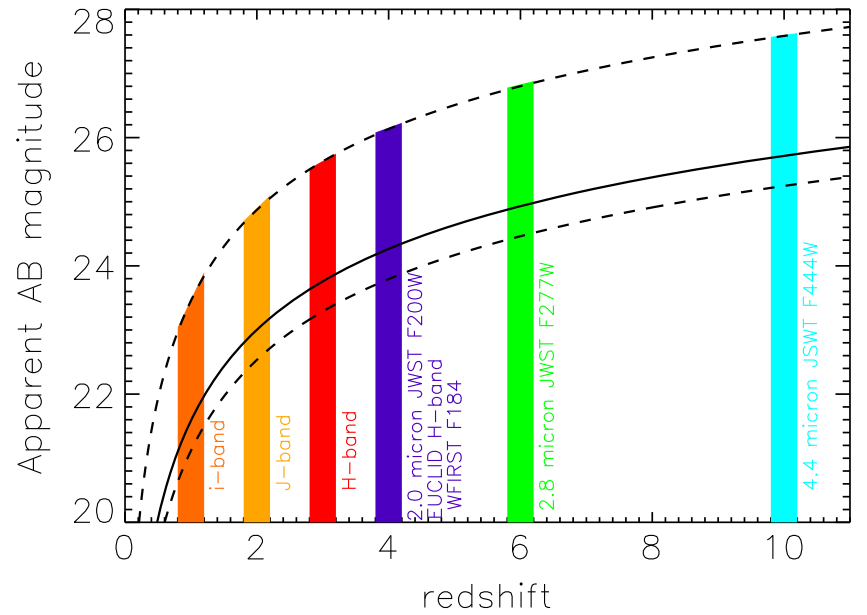

Figure 7. At higher redshift, the only possibility of observing the $400 \mathrm{~nm}$ and $520 \mathrm{~nm}$ bands is using future space-based telescopes. This figure illustrates the predicted $\mathrm{AB}$ magnitude for SLSN Ic out to $z \simeq 10$. The solid line is the peak magnitude of $M(400)=-21.70$ derived here. The lower dotted line is $1 \sigma$ scatter on the peak magnitudes and the upper dotted line illustrates the depth required to reach +30 days after peak, to apply the $\Delta M_{30}(400)$ correction discussed here (addition of the typical $1.4^{\mathrm{m}}$ decline. No foreground extinction has been applied to the estimated magnitudes, since the Milky Way foreground will be minimal at these wavelengths. 

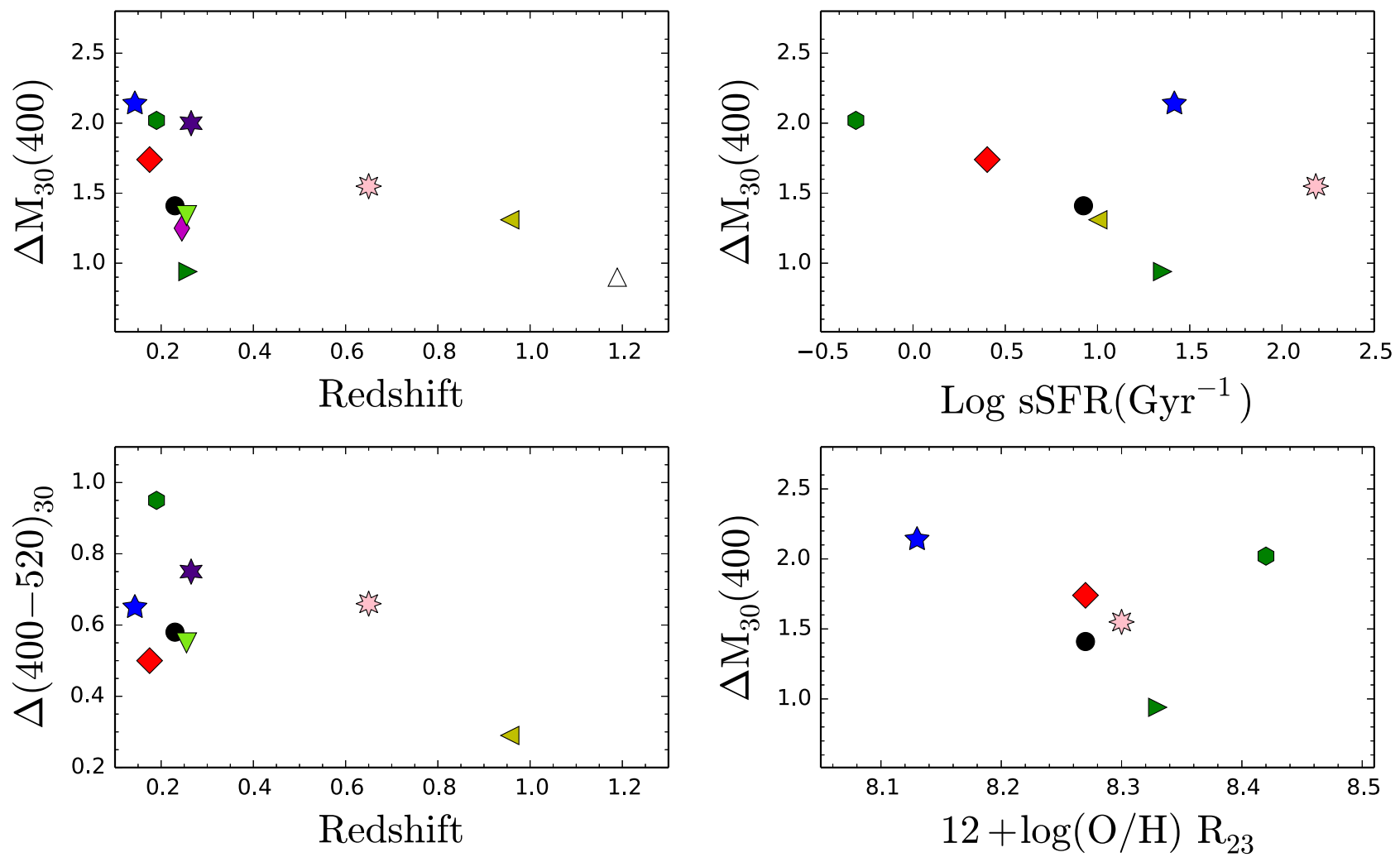

\begin{tabular}{|llll|}
$\bullet$ & SN2010gx & & PTF11rks \\
$\diamond$ & SN2011kf & $\star$ & SN2011ke \\
$\diamond$ & SN2012il & $\sum_{3}$ & PS1-10bzj \\
$\triangleleft$ & PS1-10ky & $\nabla$ & LSQ12dlf \\
$\diamond$ & PTF09cnd & $\star$ & SN2013dg \\
$\triangle$ & SCP-06F6 & & \\
\hline
\end{tabular}

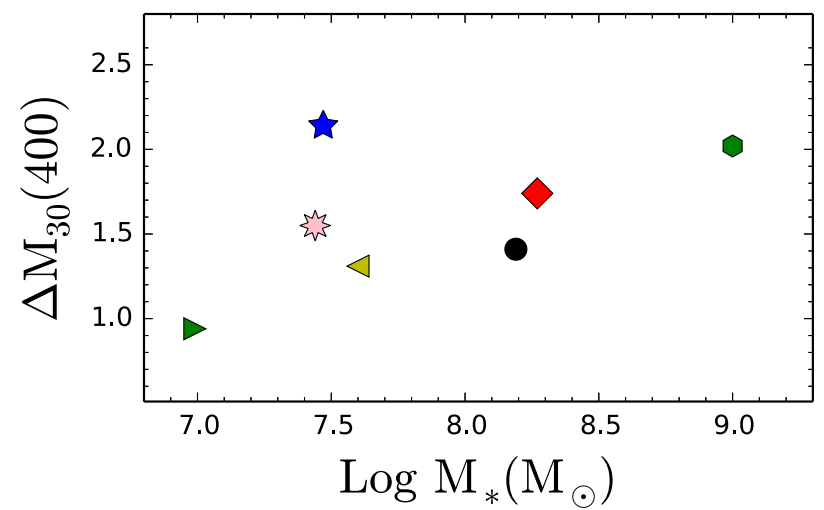

Figure 8. Various plots of example empirical properties of SLSN derived in this paper compared to redshift and host galaxy properties from Chen et al. (2013) and Lunnan et al. (2014).

We have also checked the color correlation over the 20-day evolution timescale with the updated values (Section 5 of the original paper). For $\Delta M(400-520)_{20}$ we found an rms of $0.30 \mathrm{mag}(0.29$ for the extended sample) and lower statistical coefficients than for the $\Delta M(400-520)_{30}$ (0.67 for the Spearman coefficient and 0.67 for the $r$ Pearsons coefficient).

We are very grateful to Ragnhild Lunnan for initially bringing the errors in Table 1 to our attention.

\section{REFERENCES}

Barbary, K., Dawson, K. S., Tokita, K., et al. 2009, ApJ, 690, 1358 Benetti, S., Nicholl, M., Cappellaro, E., et al. 2014, MNRAS, 441, 289 Chen, T.-W., Smartt, S. J., Bresolin, F., et al. 2013, ApJ, 763, L28 Chomiuk, L., Chornock, R., Soderberg, A. M., et al. 2011, ApJ, 743, 114 Gehrels, N. 1986, ApJ, 303, 336

Inserra, C., Smartt, S. J., Jerkstrand, A., et al. 2013, ApJ, 770, 128 Lunnan, R., Chornock, R., Berger, E., et al. 2013, ApJ, 771, 97

Lunnan, R., Chornock, R., Berger, E., et al. 2014, ApJ, 787, 138
McCrum, M., Smartt, S. J., Kotak, R., et al. 2014, MNRAS, 437, 656 McCrum, M., Smartt, S. J., Rest, A., et al. 2015, MNRAS, 448, 1206 Nicholl, M., Smartt, S. J., Jerkstrand, A., et al. 2013, Natur, 502, 346 Nicholl, M., Smartt, S. J., Jerkstrand, A., et al. 2014, MNRAS, 444, 2096 Pastorello, A., Smartt, S. J., Botticella, M. T., et al. 2010, ApJ, 724, L16 Quimby, R. M., Kulkarni, S. R., Kasliwal, M. M., et al. 2011, Natur, 474, 487

Quimby, R. M., Aldering, G., Wheeler, J. C., et al. 2007, ApJ, 668, L99 